\title{
Hybrid Green's functions for multiple cracks: Numerical extension of single crack analytical Green's functions to multiple cracks
}

\author{
M. Denda \\ Rutgers University, Mech. \& Aero. Engng. Dept. \\ Piscataway, NJ 08854-8058, U.S.A.
}

\begin{abstract}
The Green's functions for the line force and dislocation that satisfy the traction free boundary condition on the surfaces of arbitrary multiple straight cracks in the isotropic solids are obtained. We develop the hybrid Green's functions combining the analytical and numerical Green's function methods. The Green's function is split into the singular and the image terms. The crack opening displacement, represented as the continuous distribution of dislocation dipoles over each crack, serves as the source of the image term. It is proposed that the image term from each crack is further split into two parts: analytical image term for the individual single crack and the additional numerical image term caused by the presence of other cracks. Although, for the single crack problem, the analytical image term is the only image term needed to satisfy the traction free boundary condition, additional image term is needed to satisfy the boundary condition for multiple cracks. The advantage of the analytical image term is its ability to absorb high stress gradient when the singularity is located near one of the cracks. The additional image term, given in terms of the additional dislocation dipole distribution, needs only to provide small and smooth perturbation, due to the presence of other cracks, to satisfy the traction free boundary condition.
\end{abstract}

\section{Introduction}

The crack Green's function is obtained by superposing the singular field (singular term)in the homogeneous body and the crack solution (image term) with the negative of the traction loading on the crack surfaces induced by the singularity. Alternatively, the image term is represented in terms of the unknown crack opening displacements (CODs) which are determined to satisfy, when the singular solution is superposed, the traction free condition on the crack surface. For the single straight crack, the Green's functions can be obtained analytically. For multiple cracks methods have been proposed to derive the Green's functions numerically. The numerical Green's function technique of Telles $[1,2,3]$ has enabled the derivation of the Green's functions for more complex two-dimensional and threedimensional crack configurations. Telles et al. [1] have set up the hyper-singular integral equations for the crack surface loading of the image term and evaluated the integral by the numerical quadrature to determine the CODs. Denda and Quick [4] have represented the COD by the dislocation dipole distribution and evaluated the resulting singular and hyper-singular integrals analytically to es- 
tablish the whole crack singular element (WCSE) to be used as the image term. Imagine that the singularity is located near a crack. The crack surface loading, to be applied in the superposition process above, will have a sharp peak at the point closest to the singularity. In general, the $p$-type COD interpolation, such as the WCSE, cannot model such a rapid variation of traction well enough to produce the accurate image term solution.

By recalling that the image term arises from the COD of each crack we propose to split the image term of each crack into the analytical and the perturbational parts. The former is the image term solution obtained analytically by assuming other cracks are absent. The latter is the additional term, needed to satisfy the traction free boundary condition on the crack surface, to be determined numerically by the WCSE. because of the numerical determination of the latter part, it is called the numerical image term. Since for each crack the sum of the singular and the analytical image terms is zero on its surface, as shown in (4) for an example of two cracks, the sharp peak stress behavior described above for the near crack surface singularity can be effectively canceled by the analytical image term. The numerical image term, than can be modeled by the WCSE, need to deal with a small and smooth perturbation needed to satisfy the traction free boundary condition.

The proposed procedure, applied without the analytical image terms, still works as reported by Denda and Quick [4]. But the accuracy deteriorates when the singularity is located near one of the cracks. The proposed use of the analytical image terms will alleviate this situation as long as all cracks are moderately separated. However, the scheme fails if two (or more) cracks are located extremely close to each other and the singularity is placed near this close vicinity of multiple cracks. In such a case the special analytical Green's function for two (or more) cracks must be used instead of the readily available analytical Green's function for the single crack.

\section{Illustration of Hybrid Green's Functions}

We first present the concept of the hybrid Green's functions for the simplest case of two cracks. The general presentation for arbitrary number of cracks will follow. Consider an infinite body with two straight cracks, 1 and 2, where either the line force or dislocation is applied at a point $\xi$, as shown in Figure 1. Under the assumption that two cracks are not located extremely closely, we will develop the hybrid Green's functions accurate for arbitrary location of $\xi$. The traction on crack 1 consists of the singular term contribution, $S_{1}$, and the image term contributions, $\tilde{R}_{11}^{\left(\tilde{\delta}_{1}\right)}$ and $\tilde{R}_{12}^{\left(\tilde{\delta}_{2}\right)}$, where $\tilde{\delta}_{1}$ and $\tilde{\delta}_{2}$ schematically represent the crack opening displacements for two cracks. Similarly we can define the singular, $S_{2}$, and image term contributions, $\tilde{R}_{22}^{\left(\tilde{\delta}_{2}\right)}$ and $\tilde{R}_{21}^{\left(\tilde{\delta}_{1}\right)}$, for the traction on crack 2 . Note that the first index of $\tilde{R}_{i j}^{\left(\tilde{\delta}_{j}\right)}$ refers to the destination crack where the traction is evaluated while the second index refers to the source crack. In deriving the 


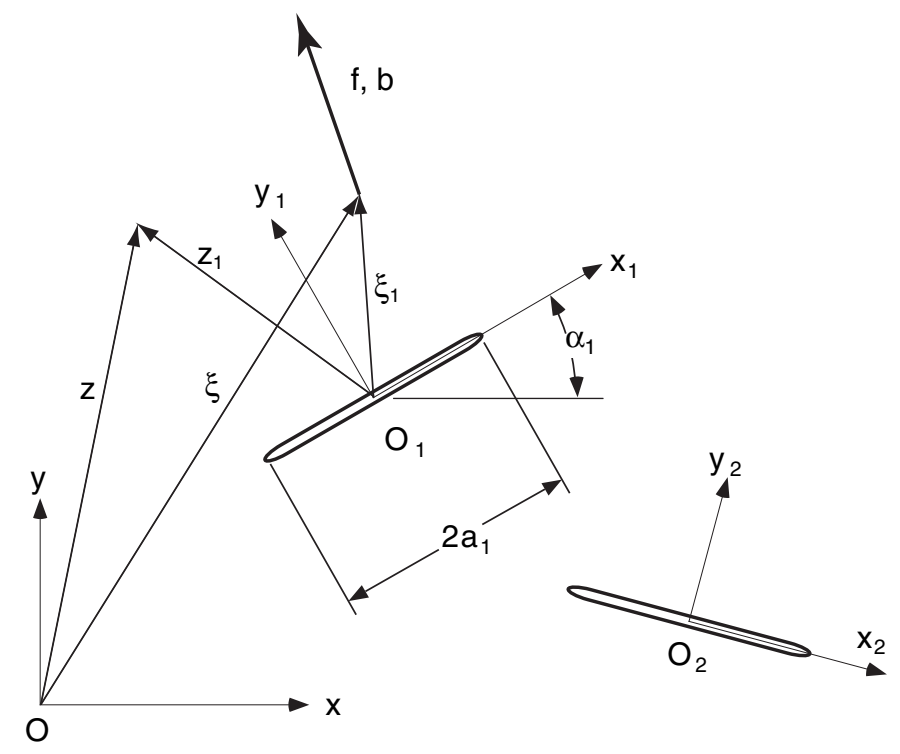

Figure 1: Local and global coordinate systems for multiple cracks.

numerical Green's functions, Denda and Quick [4] have represented the image term $\tilde{R}_{i j}^{\left(\tilde{\delta}_{j}\right)}(i, j=1,2)$ by the whole crack singular element (WCSE, explained in Appendix B) to get the zero traction equations on the crack,

$$
\begin{aligned}
& S_{1}+\tilde{R}_{11}^{\left(\tilde{\delta}_{1}\right)}+\tilde{R}_{12}^{\left(\tilde{\delta}_{2}\right)}=0, \\
& S_{2}+\tilde{R}_{21}^{\left(\tilde{\delta}_{1}\right)}+\tilde{R}_{22}^{\left(\tilde{\delta}_{2}\right)}=0,
\end{aligned}
$$

which gives the system of equations for $\tilde{\delta}_{1}$ and $\tilde{\delta}_{2}$,

$$
\begin{aligned}
& \tilde{R}_{11}^{\left(\tilde{\delta}_{1}\right)}+\tilde{R}_{12}^{\left(\tilde{\delta}_{2}\right)}=-S_{1}, \\
& \tilde{R}_{21}^{\left(\tilde{\delta}_{1}\right)}+\tilde{R}_{22}^{\left(\tilde{\delta}_{2}\right)}=-S_{2} .
\end{aligned}
$$

Once the crack opening displacements are determined solving the system (2), the numerical Green's functions are determined as linear functions of the crack opening displacements. However, when $\xi$ is very close to one of the cracks, the singular term ( $S_{1}$ or $S_{2}$ ) varies rapidly along the crack causing numerical trouble as reported by Denda and Quick [4].

To alleviate this situation split the image term $\tilde{R}_{i j}$ into two parts

$$
\tilde{R}_{i j}^{\left(\tilde{\delta}_{j}\right)}=R_{i j}^{(a n a l)}+R_{i j}^{\left(\delta_{j}\right)}
$$

where $R_{i j}^{(a n a l)}$ is the analytical image term for the single crack $j$ (assuming other crack is absent) and $R_{i j}^{\left(\delta_{j}\right)}$ is the perturbation image term due to the presence of 
the other crack. The former is obtained analytically for each crack (see Appendix A) and the latter is represented by the whole crack singular element. Note that $\tilde{\delta}_{j}$ (crack opening displacement) $\neq \delta_{j}$ (perturbed crack opening displacement). The system of equations (1) can now be written as

$$
\begin{aligned}
& S_{1}+R_{11}^{(\text {anal })}+R_{11}^{\left(\delta_{1}\right)}+R_{12}^{(\text {anal })}+R_{12}^{\left(\delta_{2}\right)}=0, \\
& S_{2}+R_{21}^{(\text {anal })}+R_{21}^{\left(\delta_{1}\right)}+R_{22}^{(\text {anal })}+R_{22}^{\left(\delta_{2}\right)}=0 .
\end{aligned}
$$

Since $S_{1}+R_{11}^{(\text {anal })}=0$ (on crack 1$)$ and $S_{2}+R_{22}^{(\text {anal })}=0$ (on crack 2 ) by definition of the analytic Green's functions, we get the system of equations

$$
\begin{aligned}
& R_{11}^{\left(\delta_{1}\right)}+R_{12}^{\left(\delta_{2}\right)}=-R_{12}^{(\text {anal })}, \\
& R_{21}^{\left(\delta_{1}\right)}+R_{22}^{\left(\delta_{2}\right)}=-R_{21}^{(\text {anal })},
\end{aligned}
$$

for the unknown perturbed crack opening displacements $\delta_{1}$ and $\delta_{2}$. Generalizing the above illustration for $N$ cracks, the corresponding system of equations can be readily obtained as

$$
\begin{aligned}
R_{11}^{\left(\delta_{1}\right)}+R_{12}^{\left(\delta_{2}\right)}+\ldots+R_{1 N}^{\left(\delta_{N}\right)}=-\left(R_{12}^{(\text {anal })}+R_{13}^{(\text {anal })}+\ldots+R_{1 N}^{(\text {anal })}\right), \\
R_{21}^{\left(\delta_{1}\right)}+R_{22}^{\left(\delta_{2}\right)}+\ldots+R_{2 N}^{\left(\delta_{N}\right)}=-\left(R_{21}^{(\text {anal })}+R_{23}^{(\text {anal })}+\ldots+R_{2 N}^{(\text {anal })}\right), \\
\vdots \\
R_{N 1}^{\left(\delta_{1}\right)}+R_{N 2}^{\left(\delta_{2}\right)}+\ldots+R_{N N}^{\left(\delta_{N}\right)}=-\left(R_{N 1}^{(\text {anal })}+R_{N 2}^{(\text {anal })}+\ldots+R_{N N}^{(\text {anal })}\right) .
\end{aligned}
$$

Once the crack opening displacement coefficients $\delta_{i}(i=1, \ldots, N)$ are determined, the hybrid Green's functions are established for the calculation of the displacement and stress fields in the domain with multiple cracks as well as the stress intensity factors for each crack tip.

\section{Implementation of Hybrid Green's Functions}

\subsection{Fundamental solutions in elasticity}

The fundamental (or singularity) solutions of elasticity consist of the line force and dislocation. The summary of derivation by Denda and Dong [5], using Muskhelishvili's complex variable formalism [6], is given below. Consider a line force $f=f_{x}+i f_{y}$ or dislocation $b=b_{x}+i b_{y}$ at $\xi$ in the infinite plane, where $b$ is Burgers vector of the dislocation. The displacement at $z=x+i y$ is given by

$$
u^{(s)}(z, \xi) \equiv u_{x}^{(s)}+i u_{y}^{(s)}=-\frac{\gamma}{2 \mu}\{\kappa \log (z-\xi)-k \overline{\log (z-\xi)}\}+\frac{\bar{\gamma}}{2 \mu} \frac{(z-\xi)}{(\overline{z-\xi})},
$$

and the stress components by

$$
\sigma^{(s)}(z, \xi) \equiv \frac{\sigma_{x x}^{(s)}+\sigma_{y y}^{(s)}}{2}=-2 \operatorname{Re}\left[\frac{\gamma}{(z-\xi)}\right]
$$




$$
\tau^{(s)}(z, \xi) \equiv \frac{\sigma_{y y}^{(s)}-\sigma_{x x}^{(s)}}{2}+i \sigma_{x y}^{(s)}=\gamma \frac{(\overline{z-\xi})}{(z-\xi)^{2}}-\bar{\gamma} \frac{k}{(z-\xi)}
$$

where

$$
\left\{\begin{array}{lll}
k=-\kappa, & \gamma=f / 2 \pi(\kappa+1) & \text { line force, } \\
k=1, & \gamma=i \mu b / \pi(\kappa+1) & \text { line dislocation, }
\end{array}\right.
$$

$\mu$ is the shear modulus and $\kappa$ is Muskhelishvili constant given by $\kappa=3-4 \nu$ in plane strain and $\kappa=(3-\nu) /(1+\nu)$ in plane stress in terms of Poisson's ratio $\nu$. The traction $t=t_{x}+i t_{y}$ at $z$ on a line segment with the slope $\theta$ is given by

$$
t^{(s)}(z, \xi ; \theta)=-i\left\{e^{i \theta} \sigma^{(s)}(z, \xi)+e^{-i \theta} \overline{\tau^{(s)}(z, \xi)}\right\}
$$

\subsection{Analytical Green's function for single crack}

We consider the line force and dislocation when the infinite domain is cracked over the interval $-a \leq x \leq a$ of the $x$-axis. As shown in Appendix A, the Green's function solutions are given in the form

$$
\begin{aligned}
& u^{(\text {total })}=u^{(s)}+u^{(\text {anal })}, \quad t^{(\text {total })}=t^{(s)}+t^{(\text {anal })}, \\
& \sigma^{(\text {total })}=\sigma^{(s)}+\sigma^{(\text {anal })}, \quad \tau^{(\text {total })}=\tau^{(s)}+\tau^{(\text {anal })},
\end{aligned}
$$

for the displacement, traction and stress components consisting of the singular terms $u^{(s)}, t^{(s)}, \sigma^{(s)}$ and $\tau^{(s)}$ and the analytical image terms $u^{(\text {anal })}, t^{(\text {anal })}, \sigma^{(\text {anal })}$ and $\tau^{(\text {anal })}$. Further details including the crack opening displacement and the stress intensity factor results are given in Appendix A.

\subsection{Whole crack singular element for single crack}

Consider a straight center crack in the infinite body and represent the crack opening displacement by the dislocation dipole distribution along the crack. Introduce a local coordinate system $O-x y$ with the coordinate origin $O$ at the crack center and the $x$-axis along the crack so that the crack lies in the interval $(-a, a)$. The local complex variable is denoted by $z=x+i y$ and the crack opening displacement by $\delta=\delta_{x}+i \delta_{y}$. If we normalize the local coordinates by $Z=z / a$ (i.e., $X=x / a$ and $Y=y / a)$, then the crack interval is reduced to $(-1,+1)$. Denda and Dong [5] have interpolated the crack opening displacement $\delta$ by

$$
\delta(X)=\sqrt{1-X^{2}} \sum_{m=1}^{M} \delta^{(m)} U_{m-1}(X)
$$

to embed the $\sqrt{r}$ COD behavior at the crack tips and integrated the dislocation dipole distribution analytically to develop the whole crack singular element (WCSE). The displacement, stress, traction and stress intensity factor formulas for the WCSE are give in Appendix B. 


\subsection{Hybrid Green's functions}

We implement the hybrid Green's functions for the system of two cracks, 1 and 2, introduced earlier (Figure 1). In addition to the global coordinate system $O-x y$, we introduce local coordinate systems $O_{1}-x_{1} y_{1}$ and $O_{2}-x_{2} y_{2}$ for two cracks. For crack 1 , the coordinate origin $O_{1}$ is located at the crack center and the $x_{1}$-axis along the crack as shown in Figure 1. The length and the slope of the crack 1 are $a_{1}$ and $\alpha_{1}$, respectively. The local coordinate system for crack 2 is introduced, similarly.

Given a line force $f=f_{x}+i f_{y}$ or dislocation $b=b_{x}+i b_{y}$ at $\xi$ in an infinite body with two cracks, the hybrid Green's functions for the displacement, stress and traction are given by

$$
\begin{aligned}
& u^{(\text {total })}(z, \xi)=u^{(s)}(z, \xi) \\
+ & \sum_{j=1}^{2} e^{i \alpha_{j}}\left\{u^{(\text {anal })}\left(w_{j}, \rho_{j}\right)+\sum_{m=1}^{M_{j}}\left(K^{(m)}\left(Z_{j}\right) \delta_{j}^{(m)}+\overline{L^{(m)}\left(Z_{j}\right) \delta_{j}^{(m)}}\right)\right\}, \\
& \sigma^{(\text {total })}(z, \xi)=\sigma^{(s)}(z, \xi) \\
+ & \sum_{j=1}^{2}\left\{\sigma^{(\text {anal })}\left(w_{j}, \rho_{j}\right)+\sum_{m=1}^{M_{j}}\left(P^{(m)}\left(Z_{j}\right) \delta_{j}^{(m)}+\overline{Q^{(m)}\left(Z_{j}\right) \delta_{j}^{(m)}}\right)\right\}, \\
& \tau^{(\text {total })}(z, \xi)=\tau^{(s)}(z, \xi) \\
+ & \sum_{j=1}^{2} e^{-2 i \alpha_{j}}\left\{\tau^{(\text {anal })}\left(w_{j}, \rho_{j}\right)+\sum_{m=1}^{M_{j}}\left(R^{(m)}\left(Z_{j}\right) \delta_{j}^{(m)}+\overline{S^{(m)}\left(Z_{j}\right) \delta_{j}^{(m)}}\right)\right\}, \\
& t^{(\text {total })}(z, \xi ; \theta)=t^{(s)}(z, \xi ; \theta) \\
+ & \sum_{j=1}^{2} e^{i \alpha_{j}}\left\{t^{(\text {anal })}\left(w_{j}, \rho_{j} ; \theta\right)+\sum_{m=1}^{M_{j}}\left(K^{*(m)}\left(Z_{j}, \theta-\alpha_{j}\right) \delta_{j}^{(m)}+\overline{L^{*(m)}\left(Z_{j}, \theta-\alpha_{j}\right) \delta_{j}^{(m)}}\right)\right\} .
\end{aligned}
$$

Notice that each Green's function consists of the singular and image terms, the latter being split into the analytic and WCSE image terms. The singular terms are given by $(7)-(10)$. The analytic image term contributions $u^{(\text {anal })}\left(w_{j}, \rho_{j}\right), \ldots$, $t^{(\text {anal })}\left(w_{j}, \rho_{j} ; \theta\right)$ for crack $j$ are calculated using the local components $f_{j}=f e^{-i \alpha_{j}}$ and $b_{j}=b e^{-i \alpha_{j}}$ of the line force and dislocation in (A.3), (A.4) and (A.8). The mapped images of $z$ and $\xi$ for each crack are defined by (A.2) and denoted by $w_{j}$ and $\rho_{j}$, respectively. The WCSE image term contributions for crack $j$ are given

in terms of the crack opening displacement coefficients $\delta_{j}^{(m)}\left(m=1, \ldots, M_{j}\right)$, in the interpolation (12), which will be determined below. All components are given in the global coordinate system to enable addition. 


\subsection{Determination of crack opening coefficients}

The unknown crack opening displacement coefficients $\delta_{j}^{(m)}\left(m=1, \ldots, M_{j}\right)$ are determined by solving the system of equations (5). In setting up the system we select $M_{1}$ and $M_{2}$ collocation points on cracks 1 and 2 so that the actual number of equations in (5) is $M_{1}+M_{2}$ instead of two. We first consider three terms $\left(R_{11}^{\left(\delta_{1}\right)}, R_{12}^{\left(\delta_{2}\right)}\right.$ and $R_{12}^{(\text {anal })}$ ) in the first of the system of equations (5) calculated at $M_{1}$ collocation points located on crack 1 , which are collectively represented as $x_{1}$ and $z_{2}=x_{2}+i y_{2}$ in the two local coordinate systems, respectively. Notice that the point on the crack 1 is represented by a pure real number $x_{1}$ in the local coordinate system $O_{1}-x_{1} y_{1}$ while it takes a complex value in the local coordinate system $O_{2}-x_{2} y_{2}$. The term $R_{11}^{\left(\delta_{1}\right)}$ is the traction on crack 1 due to the whole crack singular element (WCSE) contribution of crack 1 itself and is given , from (B.7), by

$$
R_{11}^{\left(\delta_{1}\right)}=e^{i \alpha_{1}} \frac{2 \mu}{a_{1}(\kappa+1)} \sum_{m=1}^{M_{1}} m \delta_{1}^{(m)} U_{m-1}\left(X_{1}\right),
$$

where $X_{1}=x_{1} / a_{1}$ with $x_{1}$ being the local coordinate of the point on crack 1 . Further, $M_{1}$ is the number of polynomial terms used for the crack opening displacement (COD) interpolation (12) of crack 1. The term $e^{i \alpha_{1}}$ is needed to convert the local contribution to the global coordinate system.

The term $R_{12}^{\left(\delta_{2}\right)}$ is the traction on crack 1 due to the WCSE contribution of crack 2 and is given by

$$
R_{12}^{\left(\delta_{2}\right)}=-i e^{i \alpha_{1}}\left\{\sigma^{\left(\delta_{2}\right)}\left(Z_{2}\right)+e^{-2 i\left(\alpha_{1}-\alpha_{2}\right)} \overline{\tau^{\left(\delta_{2}\right)}\left(Z_{2}\right)}\right\}
$$

where

$$
\begin{aligned}
\sigma^{\left(\delta_{2}\right)}\left(Z_{2}\right) & =\frac{2 \mu}{a_{2}(\kappa+1)} R e\left\{i \sum_{m=1}^{M_{2}} m \delta_{2}^{(m)} U^{(m-1)}\left(Z_{2}\right)\right\}, \\
\tau^{\left(\delta_{2}\right)}\left(Z_{2}\right) & =-\frac{i \mu}{a_{2}(\kappa+1)} \sum_{m=1}^{M_{2}}\left[+m\left(\delta_{2}^{(m)}+\overline{\delta_{2}^{(m)}}\right) U^{(m-1)}\left(Z_{2}\right)\right. \\
& \left.+m \delta_{2}^{(m)} \frac{Z_{2}-\overline{Z_{2}}}{Z_{2}^{2}-1}\left\{(m+1) T^{(m)}\left(Z_{2}\right)-U^{(m)}\left(Z_{2}\right)\right\}\right],
\end{aligned}
$$

obtained from (B.4). The complex valued coordinate $Z_{2}=z_{2} / a_{2}$ is the normalized coordinate of $z_{2}$. The term $R_{12}^{(\text {anal })}$ is the traction on crack 1 due to the image term of the analytical Green's function defined for the single crack 2 in the local $\mathrm{O}_{2}-x_{2} y_{2}$ coordinate system and is give by

$$
R_{12}^{(\text {anal })}=-i e^{i \alpha_{1}}\left\{\sigma^{(\text {anal })}\left(\omega_{2}, \rho_{2}\right)+e^{-2 i\left(\alpha_{1}-\alpha_{2}\right)} \overline{\tau^{(a n a l)}\left(\omega_{2}, \rho_{2}\right)}\right\},
$$

where $\sigma^{(\text {anal })}\left(\omega_{2}, \rho_{2}\right)$ and $\tau^{(\text {anal })}\left(\omega_{2}, \rho_{2}\right)$ are given by (A.4) in terms of the mapped images, $\omega_{2}$ and $\rho_{2}$ defined by (A.2), of local coordinates $z_{2}$ and $\xi_{2}$. 
The terms in the second of the system of equations (5) are calculated at $M_{2}$ collocation points located on crack 2 . They are obtained by interchanging the roles of cracks 1 and 2 in the derivation of (14), (15) and (17) and will not be repeated here. For $N$ cracks similar extension can be applied to obtain the general terms appearing in the system of equations (6). Note that each equation in (6) is evaluated at $M_{i}$ collocation points located on crack $i$ to come up with $\sum_{i=1}^{N} M_{i}$ equations needed to obtain the same number of crack opening displacement coefficients defined for each crack by (12).

\subsection{Crack opening displacement and stress intensity factors}

Once the crack opening displacement coefficients $\delta_{j}^{(m)}\left(m=1, \ldots, M_{j}\right.$ and $j=$ $1, \ldots, N)$ for all cracks are determined by solving (6), field quantities are calculated by (13) everywhere outside cracks. The crack opening displacement and the stress intensity factor for crack $j$ are obtained by adding contributions from the analytic, (A.9) and (A.12), and WCSE, (12) and (B.9), image terms to get

$$
\begin{aligned}
\delta_{j}\left(X_{j}\right) & =\delta u_{j}^{(\text {anal })}+\sqrt{1-X_{j}^{2}} \sum_{m=1}^{M_{j}} \delta_{j}^{(m)} U_{m-1}\left(X_{j}\right), \\
\left(K_{I}+i K_{I I}\right)\left( \pm a_{j}\right) & =\left(K_{I}+i K_{I I}\right)_{j}^{(\text {anal })}\left( \pm a_{j}\right) \\
& +\frac{2 \mu i}{\kappa+1} \sqrt{\frac{\pi}{a_{j}}} \sum_{m=1}^{M_{j}}( \pm 1)^{m+1} m \overline{\delta_{j}^{(m)}}
\end{aligned}
$$

where the analytic terms $\delta u_{j}^{(\text {anal })}$ and $\left(K_{I}+i K_{I I}\right)_{j}^{(a n a l)}\left( \pm a_{j}\right)$ for crack $j$ are calculated using the local components $f_{j}=f e^{-i \alpha_{j}}$ and $b_{j}=b e^{-i \alpha_{j}}$ of the line force and dislocation in (A.9) and (A.12). Note that the COD and the stress intensity factor are given in the local coordinate system aligned with the crack so that the real and imaginary terms of the COD indicate the tangential and normal opening components and $K_{I}$ and $K_{I I}$ indicate MODE I and II stress intensity factors.

\section{Numerical Results}

\subsection{Normalization}

Let $q$ and $q_{0}$ represent a dimensional quantity and its reference value, respectively; its normalization is given by $\tilde{q}=q / q_{0}$. We select the reference values for the stress and strain to be $\sigma_{0}=10^{8}\left(\mathrm{~N} / \mathrm{m}^{2}\right)$ and $\epsilon_{0}=10^{-3}$, respectively. The reference values of other quantities are determined in terms of these two reference variables such that all the normalized governing equations remain exactly the same form as the original equations listed in Section 3. For example, the reference values of the stiffness and displacement are given by $c_{0}=\sigma_{0} / \epsilon_{0}=10^{11}\left(\mathrm{~N} / \mathrm{m}^{2}\right)$ and $u_{0}=$ $x_{0} \epsilon_{0}=10^{-3} x_{0}$, where $x_{0}$ is the characteristic length of the problem. Further, the 
reference values of the line force and line dislocation are given, through (7) and (9), by $f_{0}=x_{0} \sigma_{0}=10^{8} x_{0}(\mathrm{~N} / \mathrm{m})$ and $b_{0}=x_{0} \epsilon_{0}=10^{-3} x_{0}(\mathrm{~m})$. Finally, the reference value of the stress intensity factor is given by $K_{0}=\sigma_{0} \sqrt{x_{0}}=10^{8} \sqrt{x_{0}}\left(\mathrm{~N} / \mathrm{m}^{3 / 2}\right)$. For the problems considered below, we select $x_{0}$ to be the minimum half crack length $a$ among $N$ cracks considered, i.e., $x_{0}=a$. In summary, in the nondimensional coordinate system given by

$$
\tilde{x}=\frac{x}{a}, \quad \tilde{y}=\frac{y}{a},
$$

we specify the non-dimensional line force and line dislocation,

$$
\tilde{f}=\frac{f(N / m)}{10^{8} a(N / m)}, \quad \tilde{b}=\frac{b(m)}{10^{-3} a(m)},
$$

to calculate the resulting non-dimensional displacement and stress,

$$
\tilde{u}=\frac{u(m)}{10^{-3} a(m)}, \quad \tilde{\sigma}=\frac{\sigma\left(N / m^{2}\right)}{10^{8}\left(N / m^{2}\right)} .
$$

The non-dimensional shear modulus is given by

$$
\tilde{\mu}=\frac{\mu\left(N / m^{2}\right)}{10^{11}\left(N / m^{2}\right)}
$$

and the non-dimensional crack opening coefficients for the WCSE image terms in (12) are given by

$$
\tilde{\delta}_{j}^{(m)}=\frac{\delta_{j}^{(m)}(m)}{10^{-3} a(m)} .
$$

The non-dimensional stress intensity factors are given by

$$
\tilde{K}_{I}+i \tilde{K}_{I I}=\frac{K_{I}+i K_{I I}\left(N / m^{3 / 2}\right)}{10^{8} \sqrt{a}\left(N / m^{3 / 2}\right)} .
$$

According to the definition, all equations in Section 3 are interpreted to be the normalized (or non-dimensional) equations if all the quantities are replaced by their normalized quantities. Numerical results are obtained for such normalized quantities below.

\subsection{Comparison of analytical and numerical Green's func- tions for a single crack}

For the single center crack the hybrid Green's functions are reduced to the analytic green's functions. This is the simplest crack configuration that provides an excellent test case for the performance of the hybrid Green's functions. We have calculated the stress intensity factors (SIFs) by the analytical and numerical Green's functions and obtained the relative error in the SIFs as the function of 

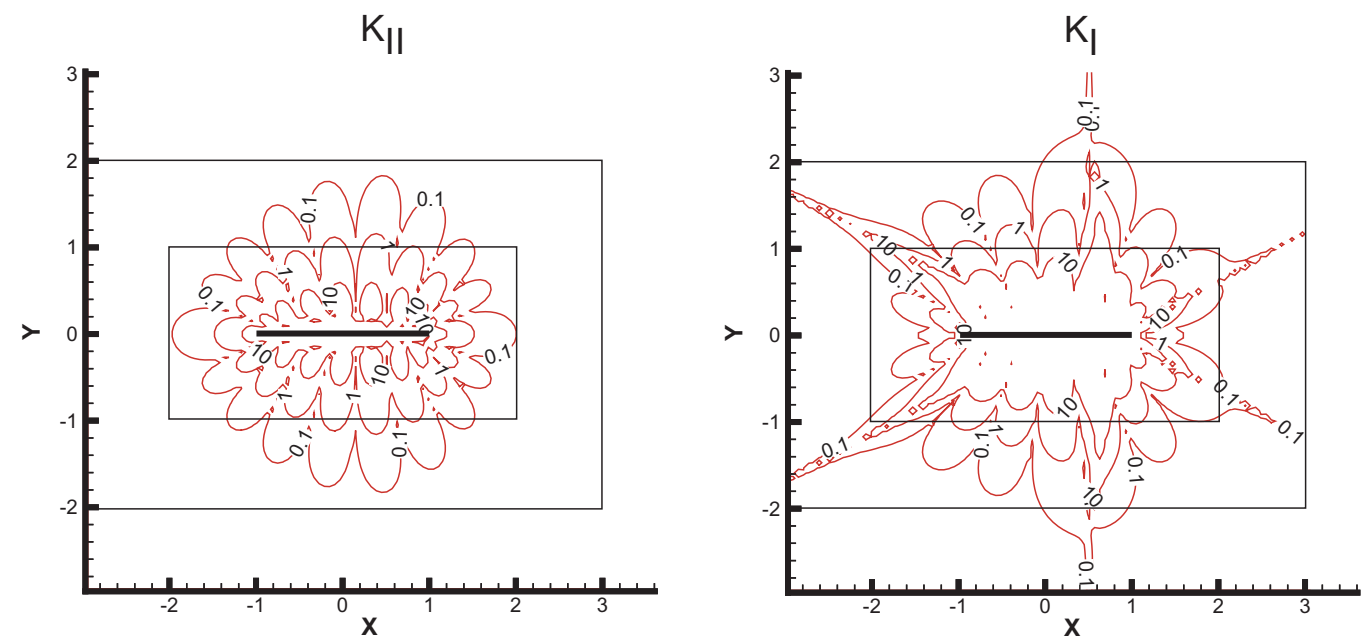

(a)
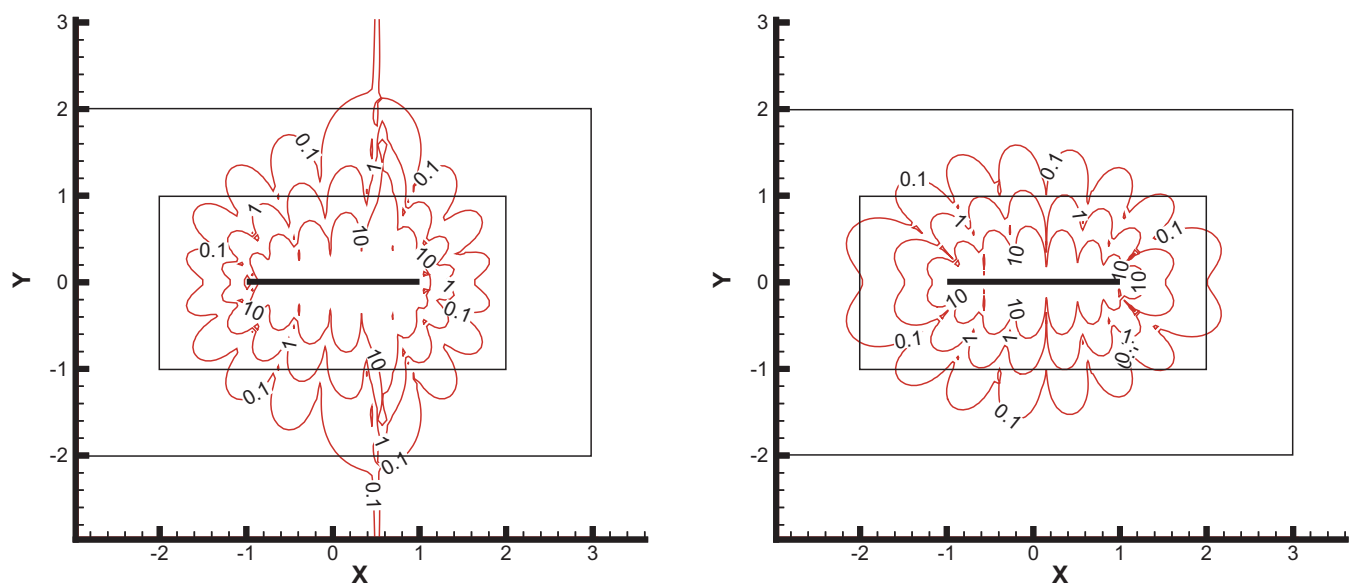

(b)

Figure 2: Variation of the relative error (in \%) in $K_{I}(+1)$ and $K_{I I}(+1)$ as the function of the location $(x, y)$ of the unit line force (a) $f_{x}$ and (b) $f_{y}$. Crack lies in the interval $(-1,+1)$ on the $x$ - axis. Number of polynomials used is $M=7$. 

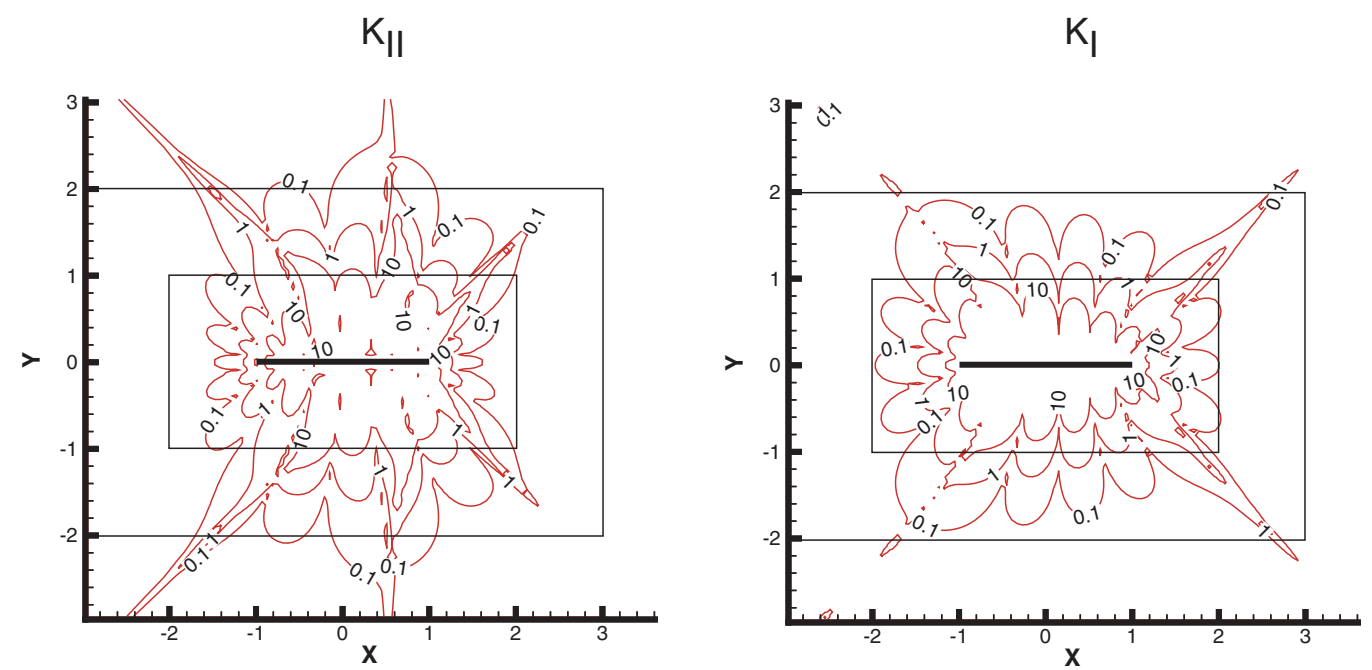

(a)
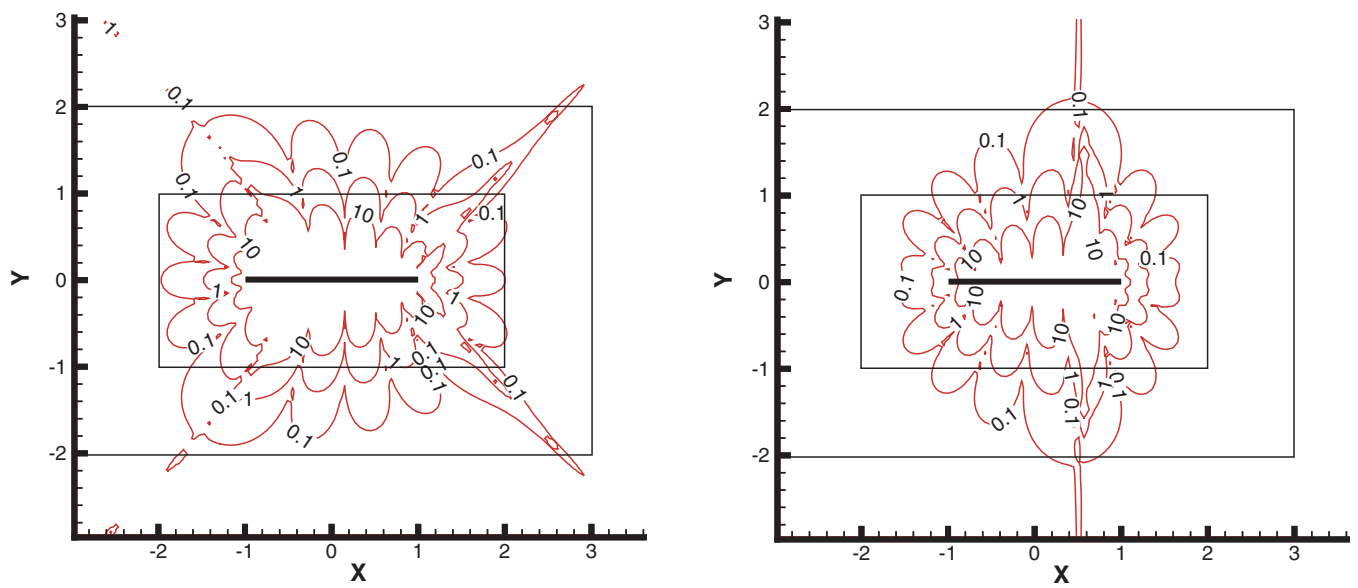

(b)

Figure 3: Variation of the relative error (in \%) in $K_{I}(+1)$ and $K_{I I}(+1)$ as the function of the location $(x, y)$ of the unit line force (a) $b_{x}$ and (b) $b_{y}$. Crack lies in the interval $(-1,+1)$ on the $x$ - axis. Number of polynomials used is $M=7$. 


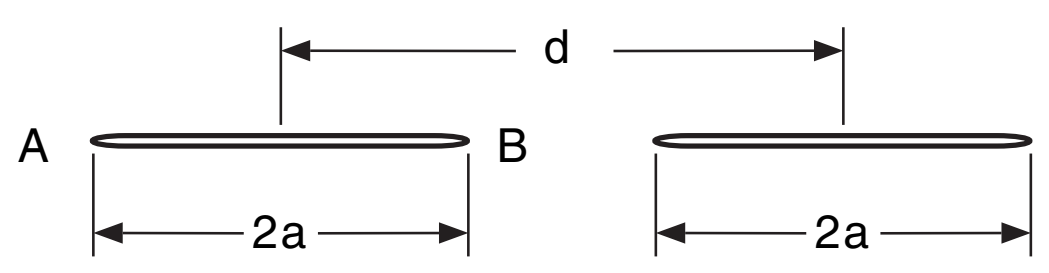

Figure 4: Two collinear cracks with $d=4 a$.

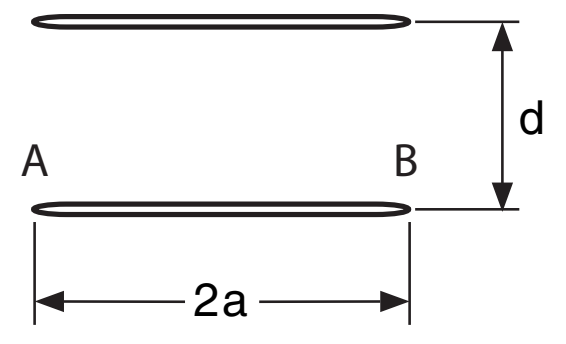

Figure 5: Two parallel cracks with $d=2 a$.

the location for the fundamental solutions consisting of the line force and dislocation. The non-dimensional shear modulus and Poisson's ratio are $\tilde{\mu}=1.0$ and $\nu=0.3$, respectively. Plane strain is assumed. The number of the terms used in the interpolation (12) for the numerical Green's functions is $M=7$. Each plot in the Figures 2 and 3 has three contours marking the boundaries of $0.1 \%, 1 \%$ and $10 \%$ relative errors, respectively. These figures show that the error introduced by the numerical Green's functions for the fundamental solutions near the crack is effectively removed by the use of the analytical Green's functions. This effective removal of error extends to multiple crack configurations modeled by the hybrid Green's functions in the same fashion as the single crack case.

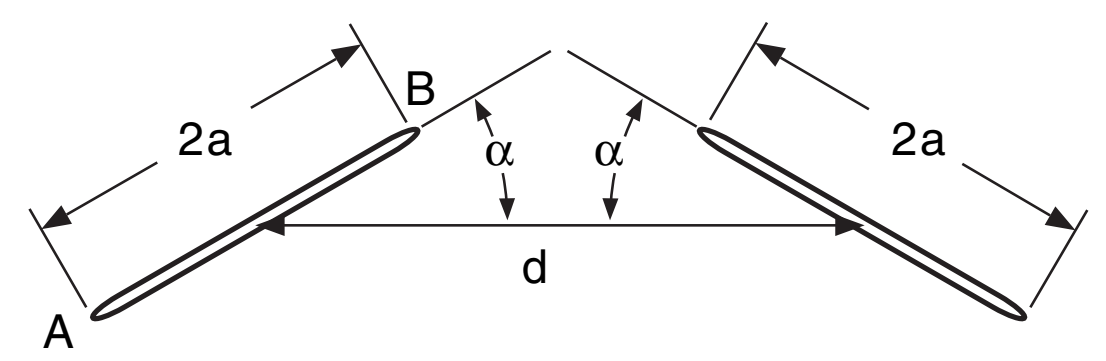

Figure 6: Two inclined cracks with $d=2 a$ and $\alpha=45^{\circ}$. 


\subsection{Stress intensity factors for multiple cracks}

Figures 4-6 show three crack configurations, two collinear, two parallel, and two inclined cracks, for which the hybrid Green's functions are obtained. The non-dimensional shear modulus and Poisson's ratio are $\tilde{\mu}=1.0$ and $\nu=0.3$, respectively. Plane strain is assumed.

We have placed the unit line force components $\tilde{f}_{x}$ and $\tilde{f}_{y}$ and line dislocation components $\tilde{b}_{x}$ and $\tilde{b}_{y}$, respectively, at the location $(\tilde{x}, \tilde{y})$ to calculate the resulting stress intensity factors (SIFs), $\tilde{K}_{I}$ and $\tilde{K}_{I I}$, at one of the crack tips. By changing the location we have calculated the SIFs as the function of the location and plotted in Figures 7 - 16. Note that all results are given in terms of the normalized quantities introduced earlier. The characteristic length for each crack problem is given by $x_{0}=a$.

Figure 17 shows 3-D plots of $\tilde{u}_{y}$ displacement field for the two parallel crack subjected to $\tilde{f}_{y}=1.0$ located on the upper lip at the middle of the lower crack $A B$. Notice the perfect shape of the crack opening displacement obtained by the hybrid Green's function, which is impossible by the simple numerical Green's function. Similar accurate results have been obtained for the other displacement and stress components due to other line force and dislocation components for all crack configurations considered.

\section{Conclusion}

We have developed the hybrid Green's functions for multiple straight crack problems combining the analytical and numerical Green's function methods. The Green's function is split into the singular and the image terms and the image term from each crack is further split into two parts: analytical image term for the individual single crack and the additional numerical image term caused by the presence of other cracks. The crack opening displacement contribution for the numerical image term is represented by the WCSE as the continuous distribution of dislocation dipoles over each crack. The analytical image term has the ability to absorb high stress gradient when the singularity is located near one of the cracks. The numerical image term, given in terms of the additional dislocation dipole distribution, needs only to provide small and smooth perturbation, due to the presence of other cracks, to satisfy the traction free boundary condition. The proposed hybrid Green's functions can accurately represent the behavior of the fundamental solutions near one of the multiple cracks that give rise to steep variation of the crack surface traction.

\section{Acknowledgment}

The research was partially supported by the NSF IGERT Grant under subcontract from the University of Tennessee, Knoxville. 


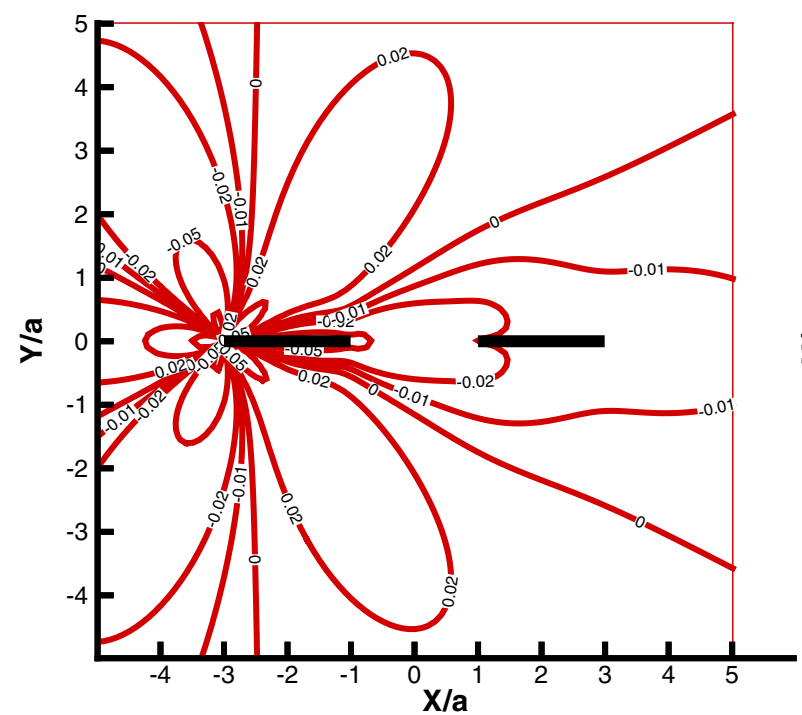

(a)

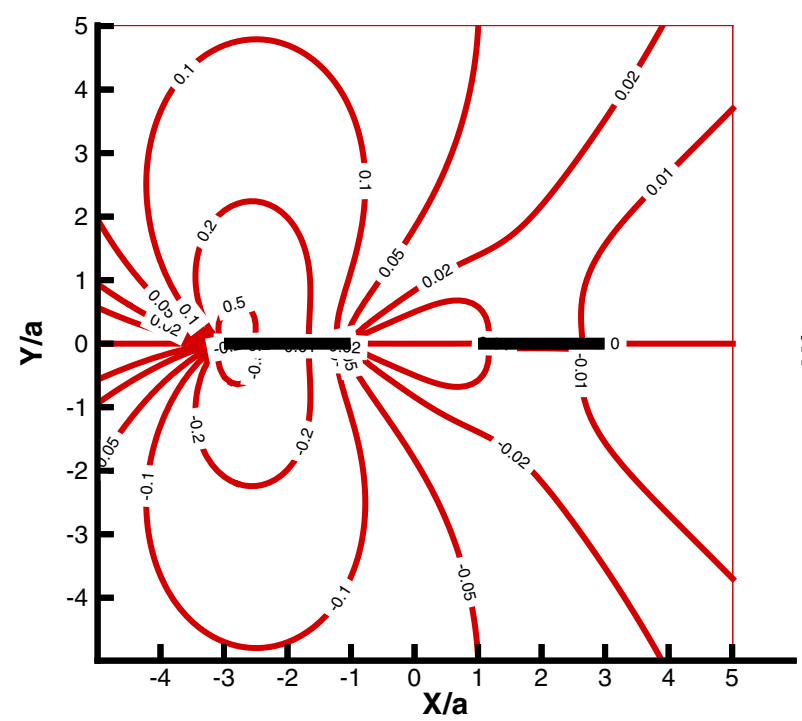

(c)

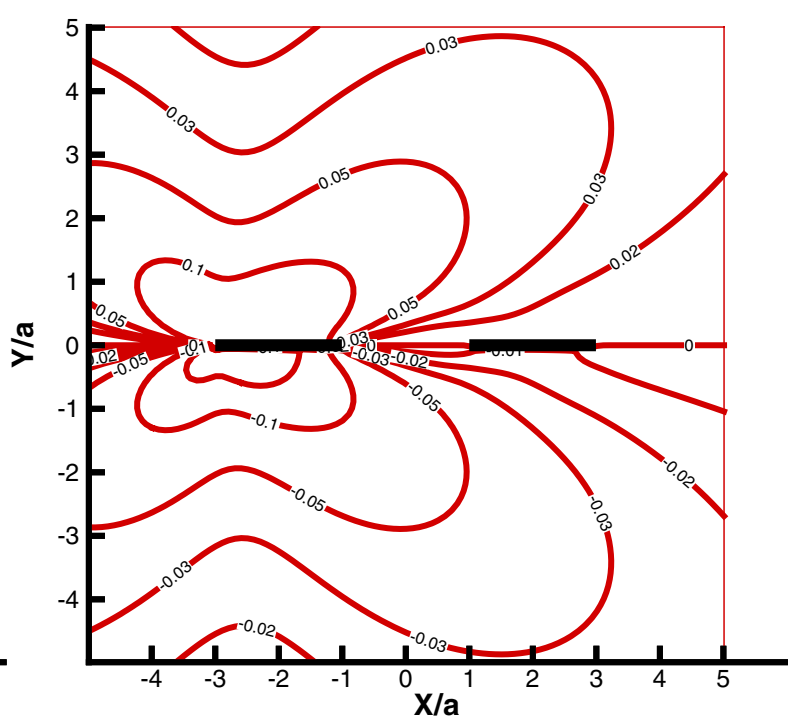

(b)

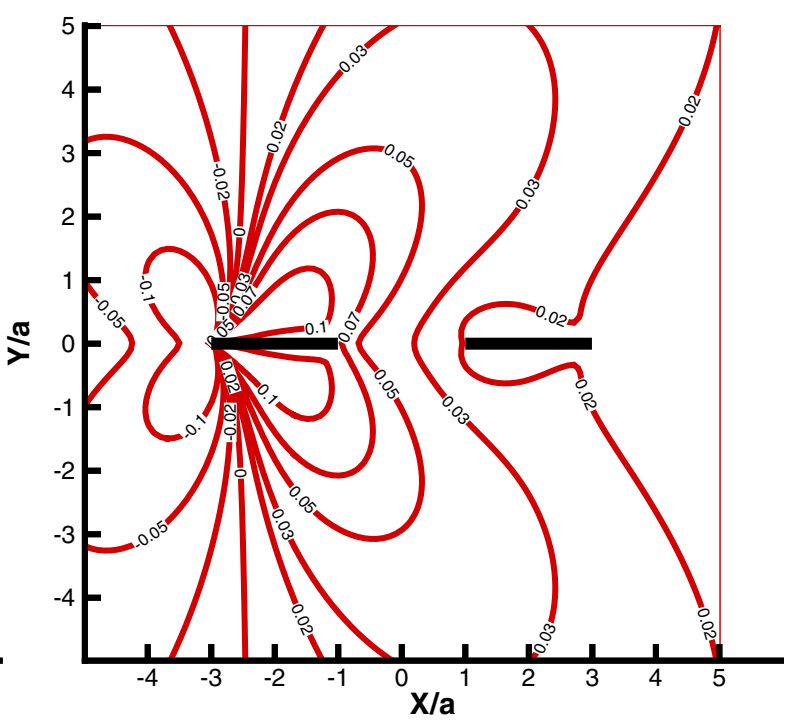

(d)

Figure 7: Variation of the SIFs as the function of the location $(\tilde{x}=x / a, \tilde{y}=y / a)$ of the unit line forces for two collinear cracks. The SIFs are measured at the crack tip $A$ in Figure 4 . (a) $\tilde{K}_{I}$ by $\tilde{f}_{x}=1$, (b) $\tilde{K}_{I I}$ by $\tilde{f}_{x}=1$, (c) $\tilde{K}_{I}$ by $\tilde{f}_{y}=1$, (b) $\tilde{K}_{I I}$ by $\tilde{f}_{x}=1$. 


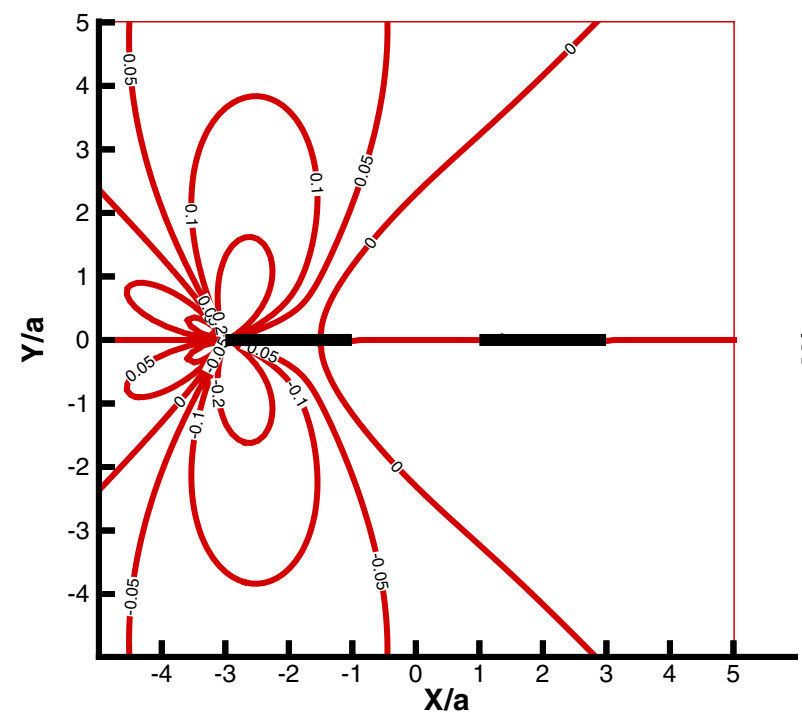

(a)

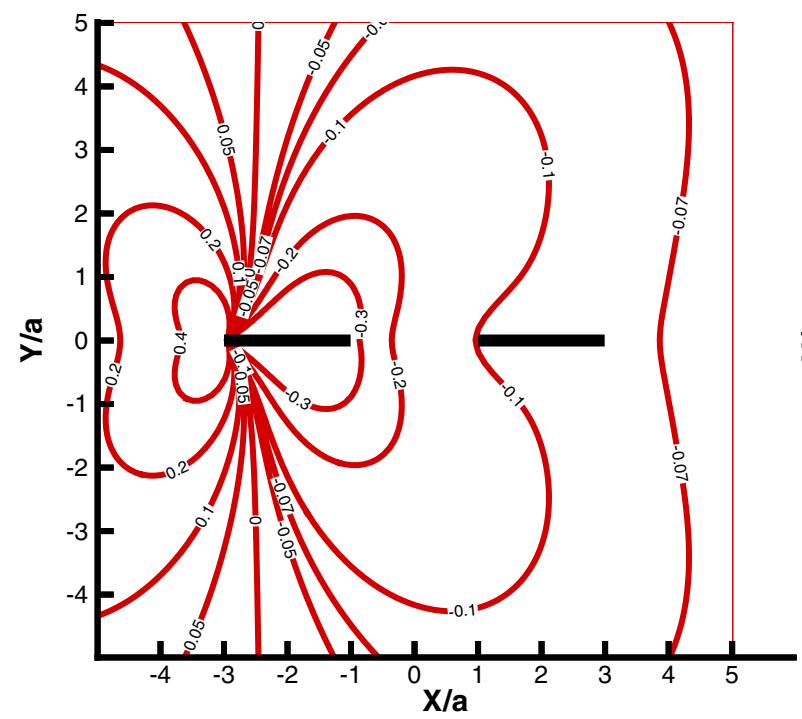

(c)

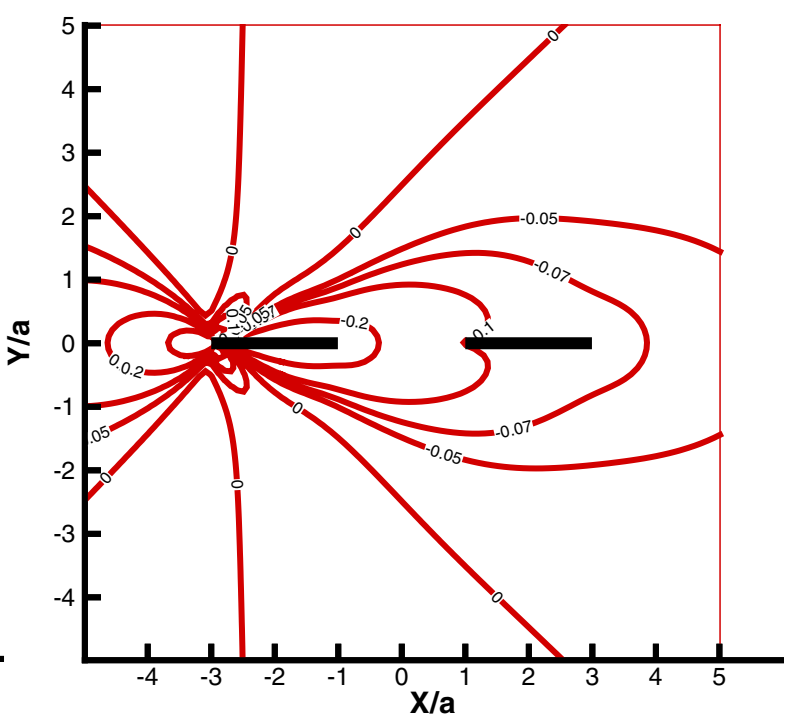

(b)

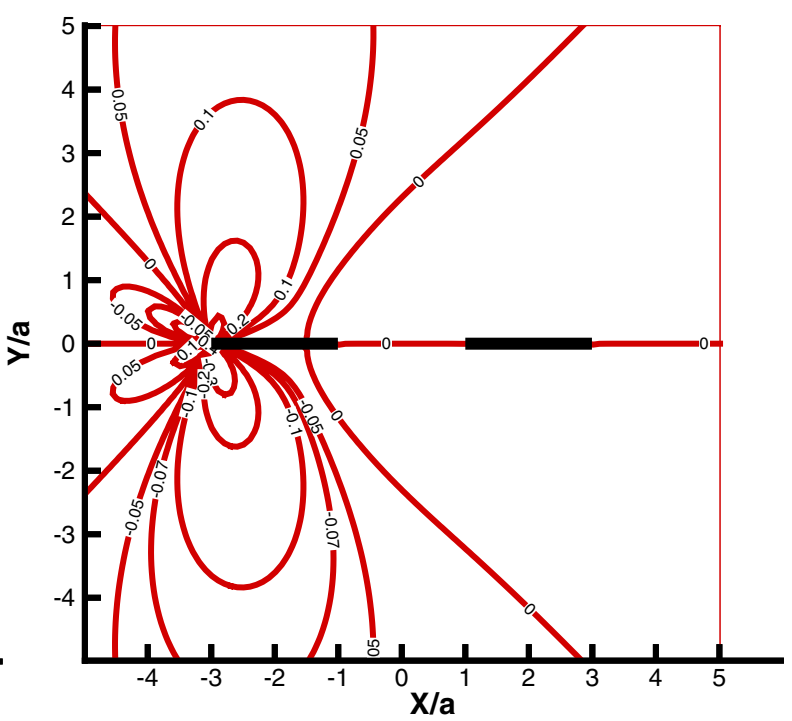

(d)

Figure 8: Variation of the SIFs as the function of the location $(\tilde{x}=x / a, \tilde{y}=y / a)$ of the unit line forces for two collinear cracks. The SIFs are measured at the crack tip $A$ in Figure 4. (a) $\tilde{K}_{I}$ by $\tilde{b}_{x}=1$, (b) $\tilde{K}_{I I}$ by $\tilde{b}_{x}=1$, (c) $\tilde{K}_{I}$ by $\tilde{b}_{y}=1$, (b) $\tilde{K}_{I I}$ by $\tilde{b}_{x}=1$. 


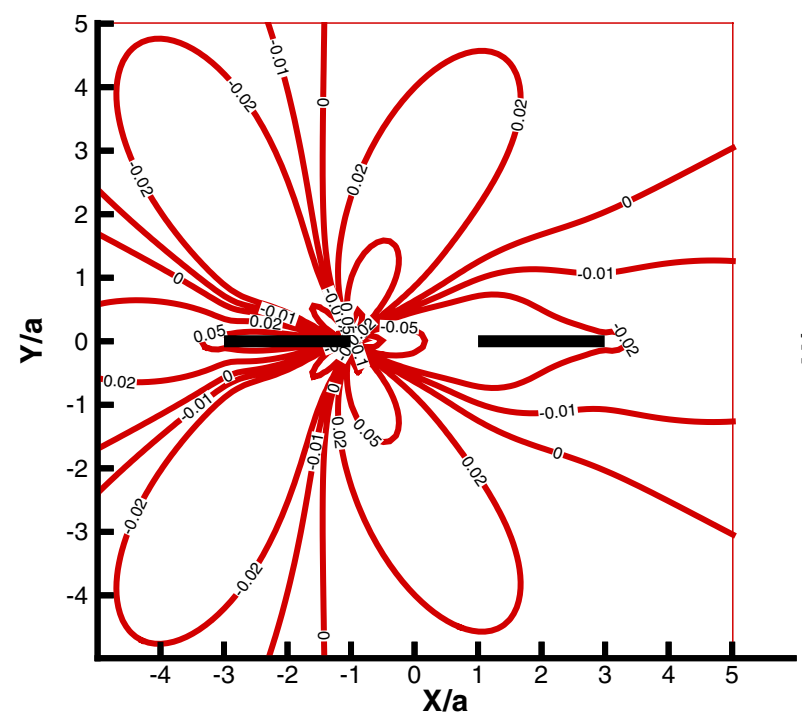

(a)

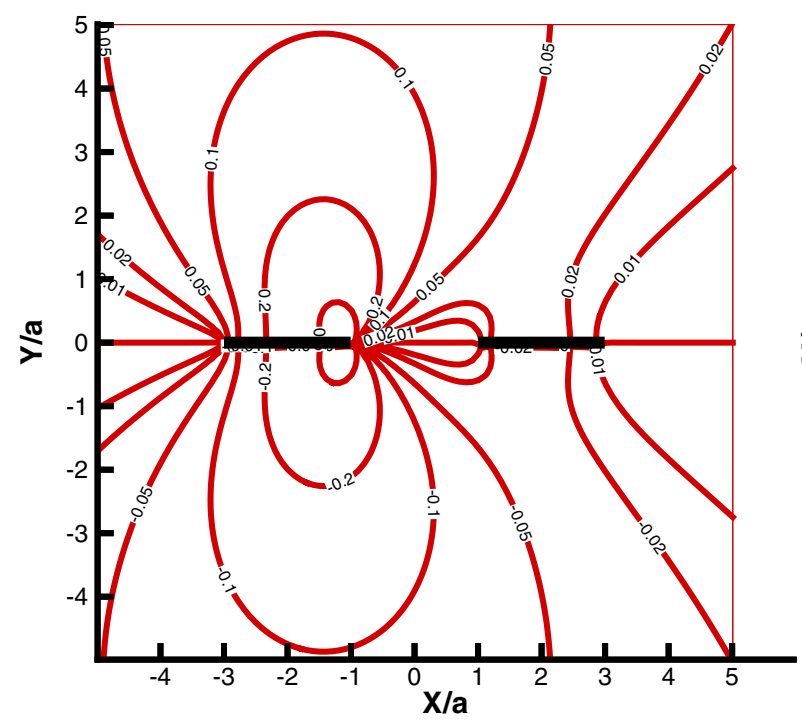

(c)

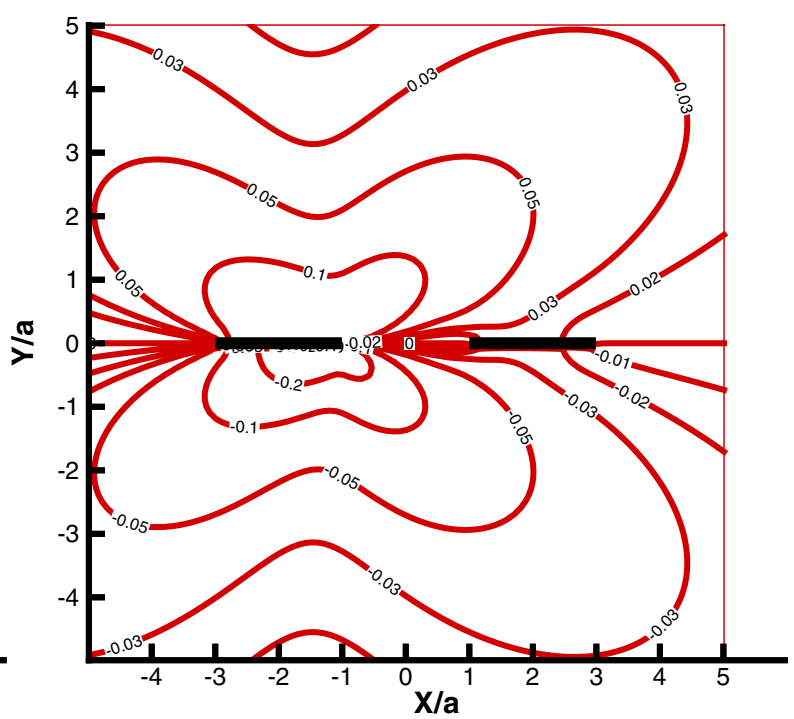

(b)

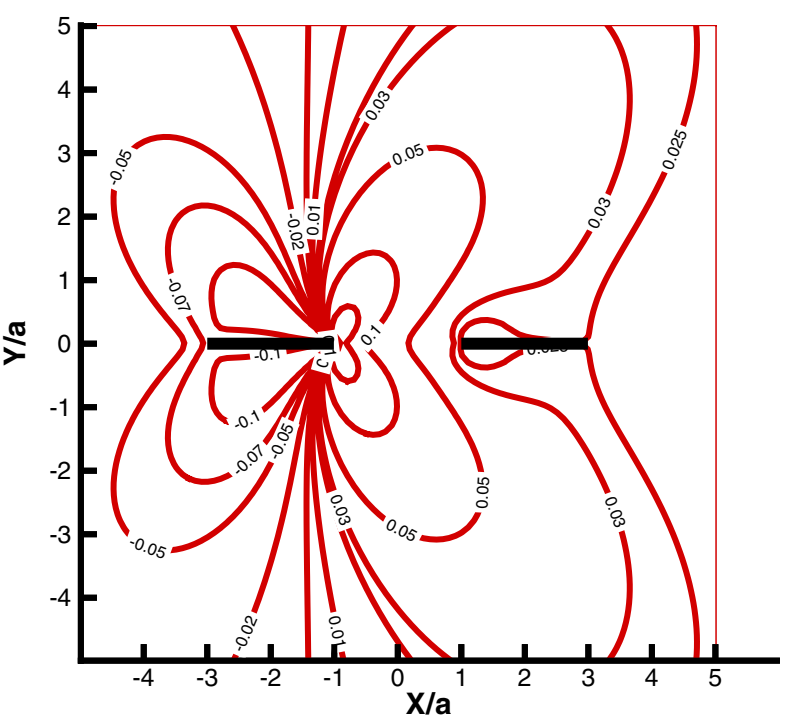

(d)

Figure 9: Variation of the SIFs as the function of the location $(\tilde{x}=x / a, \tilde{y}=y / a)$ of the unit line forces for two collinear cracks. The SIFs are measured at the crack tip $B$ in Figure 4 . (a) $\tilde{K}_{I}$ by $\tilde{f}_{x}=1$, (b) $\tilde{K}_{I I}$ by $\tilde{f}_{x}=1$, (c) $\tilde{K}_{I}$ by $\tilde{f}_{y}=1$, (b) $\tilde{K}_{I I}$ by $\tilde{f}_{x}=1$. 


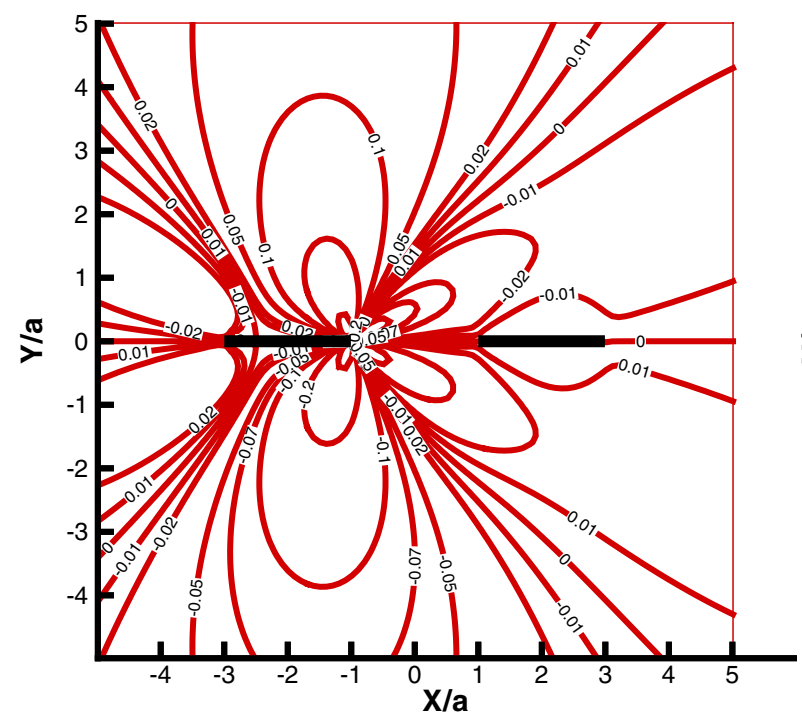

(a)

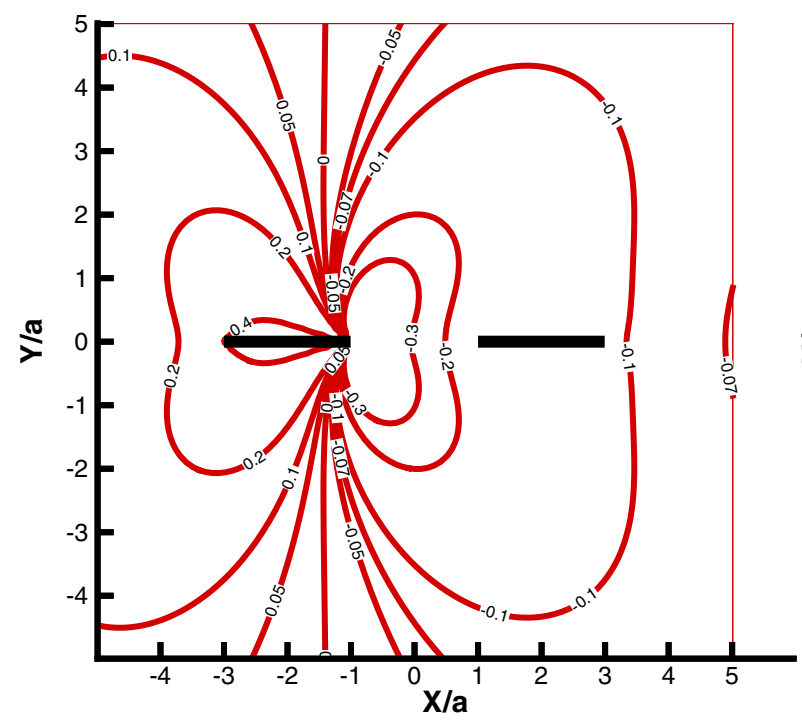

(c)

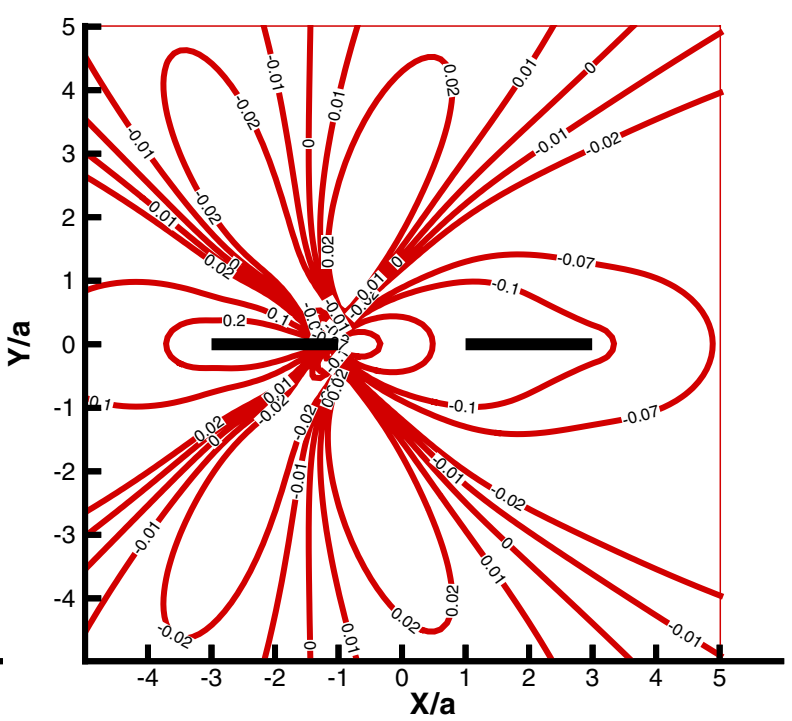

(b)

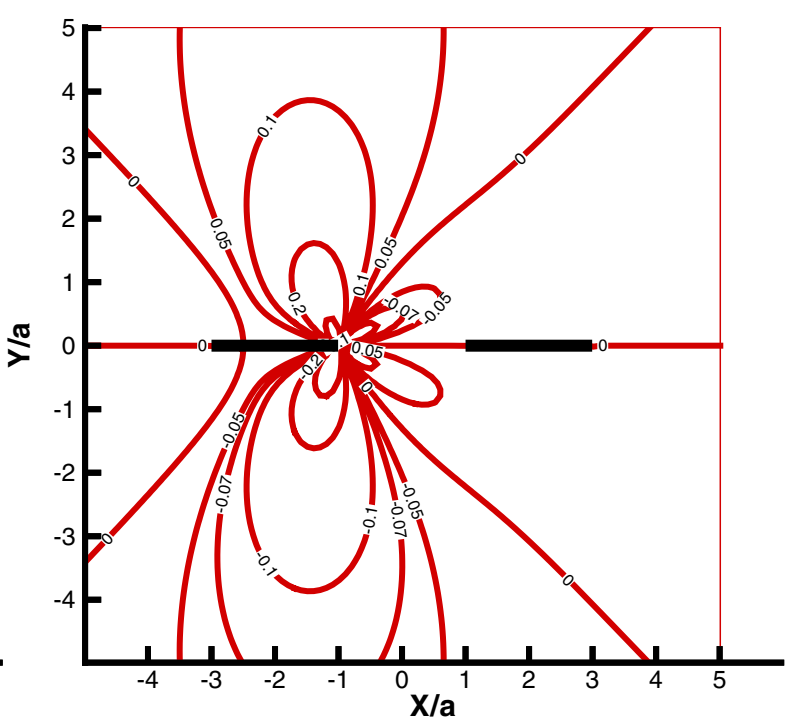

(d)

Figure 10: Variation of the SIFs as the function of the location $(\tilde{x}=x / a, \tilde{y}=y / a)$ of the unit line forces for two collinear cracks. The SIFs are measured at the crack tip $B$ in Figure 4 . (a) $\tilde{K}_{I}$ by $\tilde{b}_{x}=1$, (b) $\tilde{K}_{I I}$ by $\tilde{b}_{x}=1$, (c) $\tilde{K}_{I}$ by $\tilde{b}_{y}=1$, (b) $\tilde{K}_{I I}$ by $\tilde{b}_{x}=1$. 


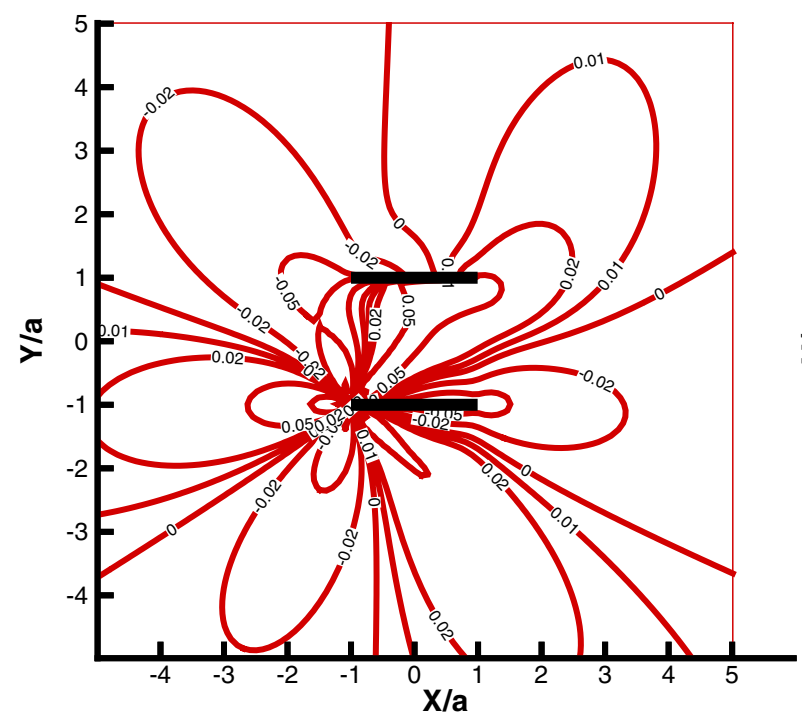

(a)

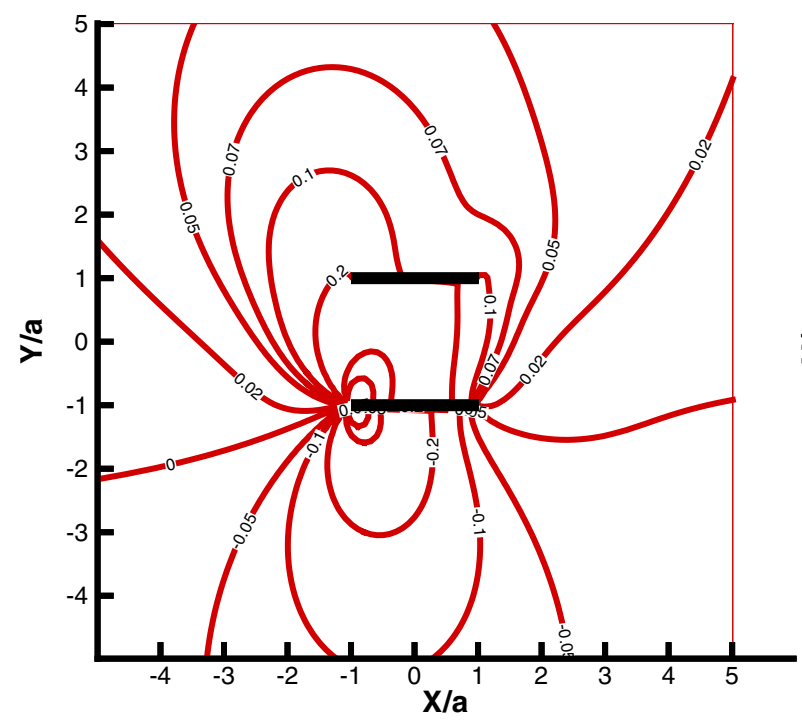

(c)

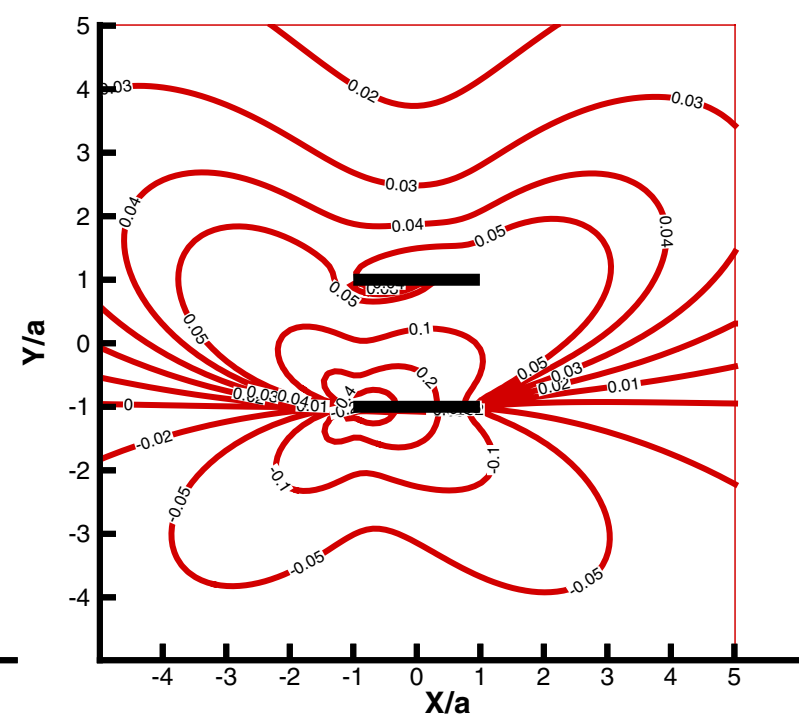

(b)

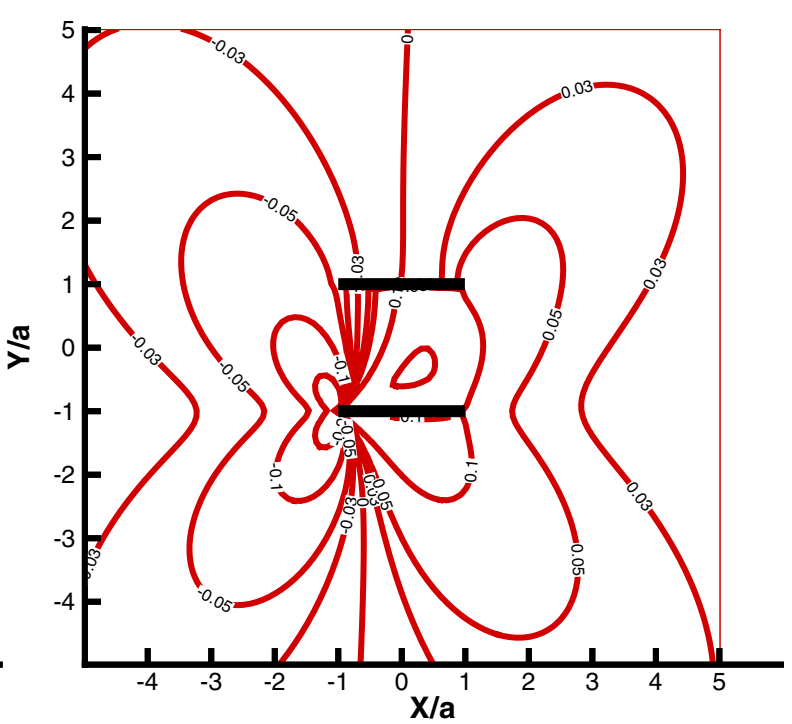

(d)

Figure 11: Variation of the SIFs as the function of the location $(\tilde{x}=x / a, \tilde{y}=y / a)$ of the unit line forces for two parallel cracks. The SIFs are measured at the crack tip $A$ in Figure 5 . (a) $\tilde{K}_{I}$ by $\tilde{f}_{x}=1$, (b) $\tilde{K}_{I I}$ by $\tilde{f}_{x}=1$, (c) $\tilde{K}_{I}$ by $\tilde{f}_{y}=1$, (b) $\tilde{K}_{I I}$ by $\tilde{f}_{x}=1$. 


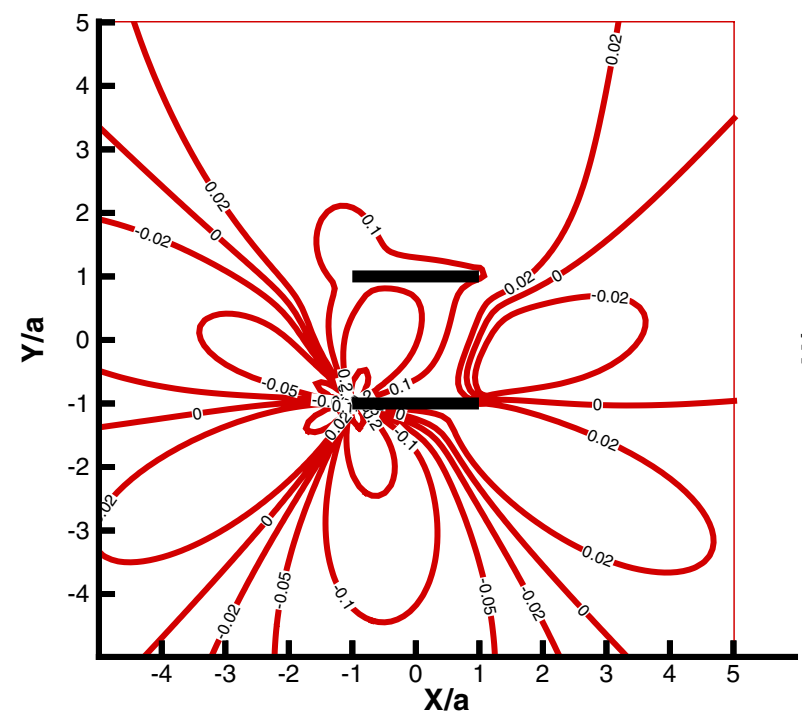

(a)

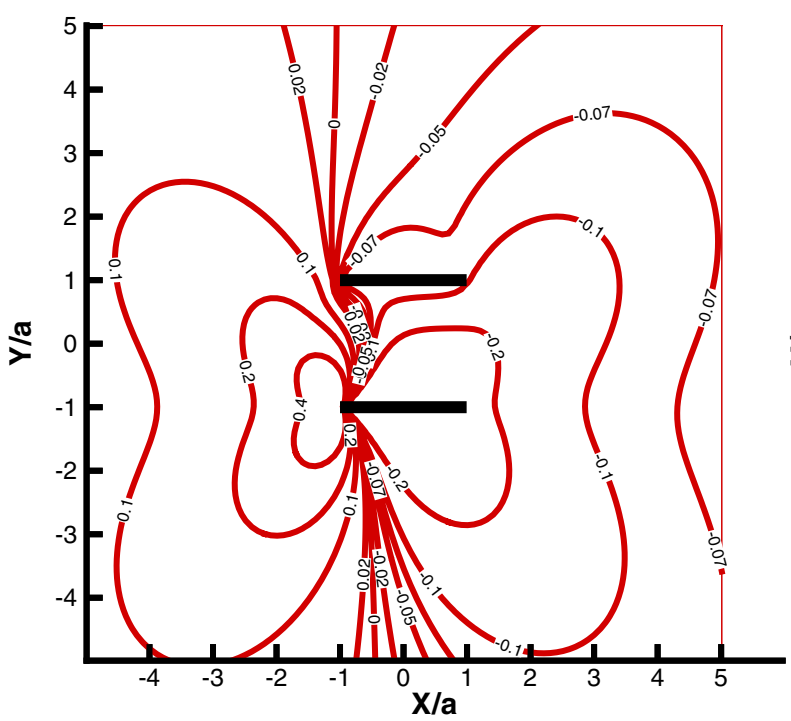

(c)

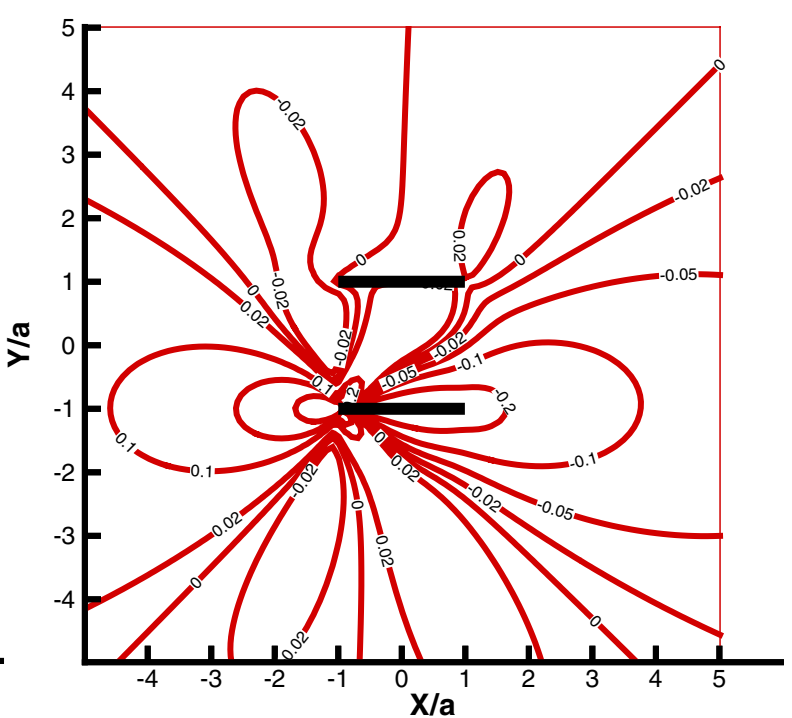

(b)

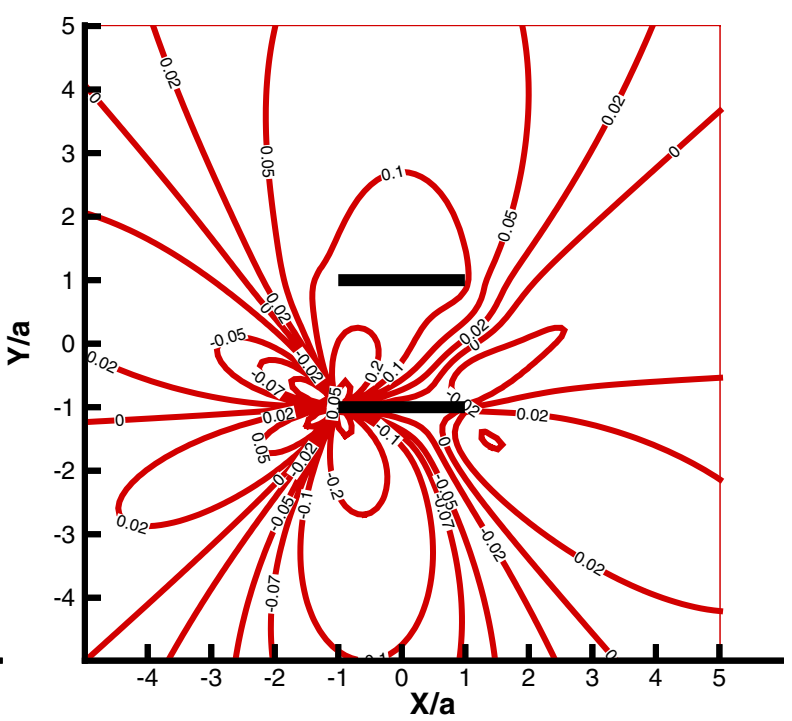

(d)

Figure 12: Variation of the SIFs as the function of the location $(\tilde{x}=x / a, \tilde{y}=y / a)$ of the unit line forces for two parallel cracks. The SIFs are measured at the crack tip $A$ in Figure 5 . (a) $\tilde{K}_{I}$ by $\tilde{b}_{x}=1$, (b) $\tilde{K}_{I I}$ by $\tilde{b}_{x}=1$, (c) $\tilde{K}_{I}$ by $\tilde{b}_{y}=1$, (b) $\tilde{K}_{I I}$ by $\tilde{b}_{x}=1$. 


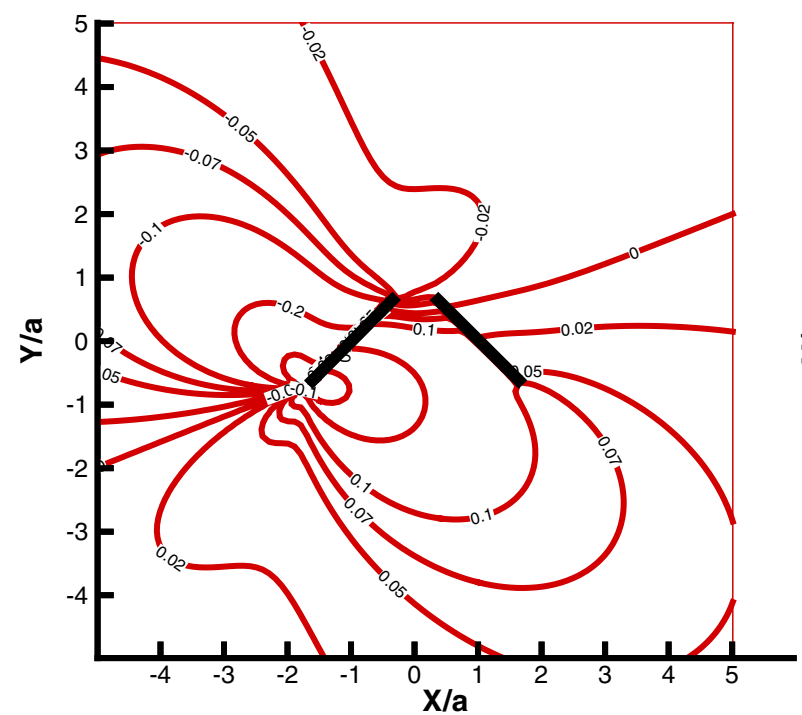

(a)

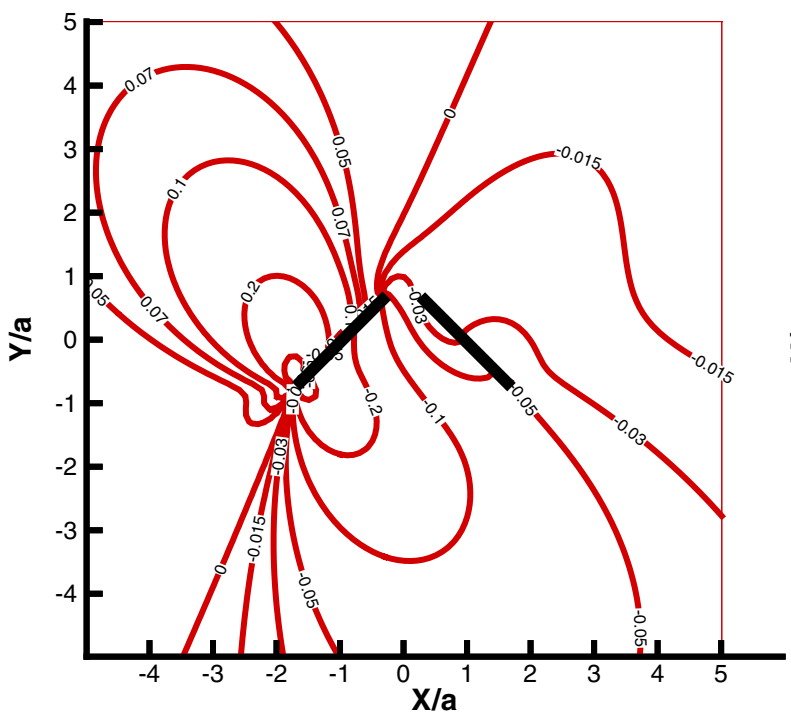

(c)

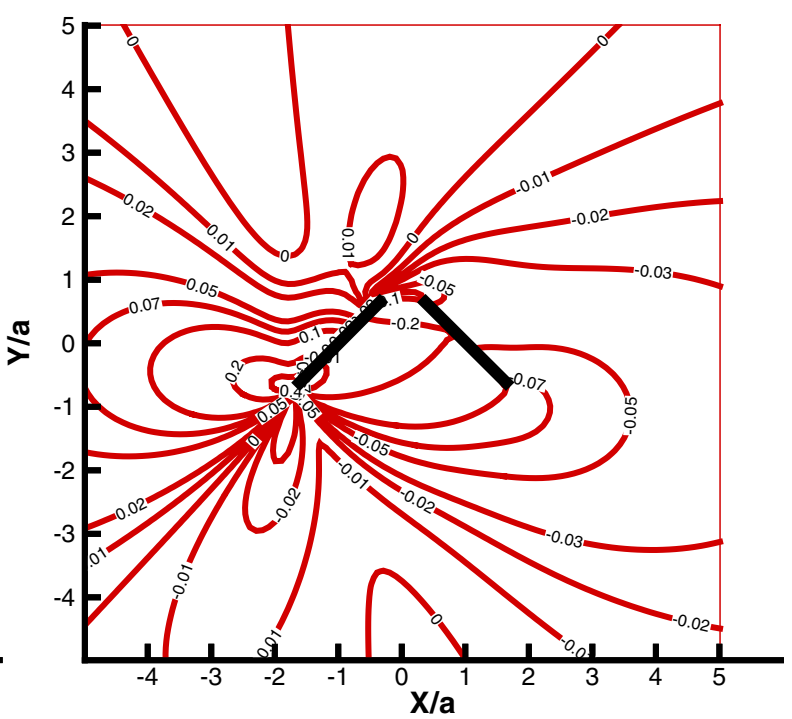

(b)

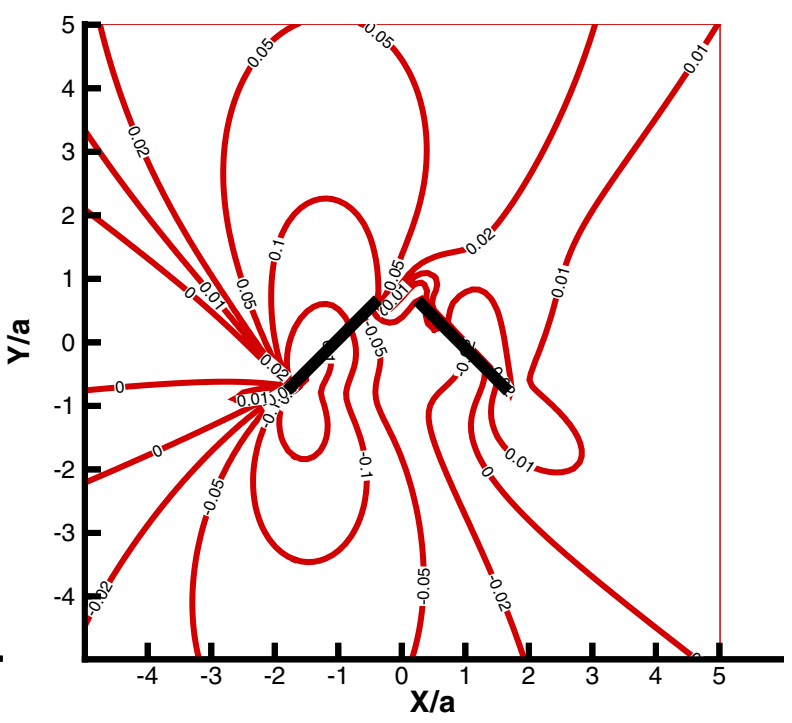

(d)

Figure 13: Variation of the SIFs as the function of the location $(\tilde{x}=x / a, \tilde{y}=y / a)$ of the unit line forces for two inclined cracks. The SIFs are measured at the crack tip $A$ in Figure 6 . (a) $\tilde{K}_{I}$ by $\tilde{f}_{x}=1$, (b) $\tilde{K}_{I I}$ by $\tilde{f}_{x}=1$, (c) $\tilde{K}_{I}$ by $\tilde{f}_{y}=1$, (b) $\tilde{K}_{I I}$ by $\tilde{f}_{x}=1$. 


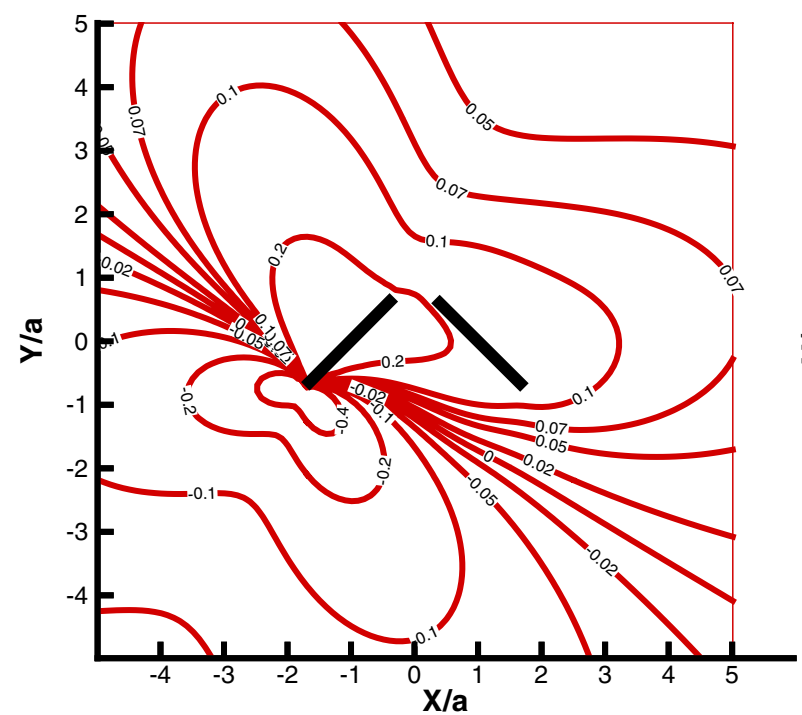

(a)

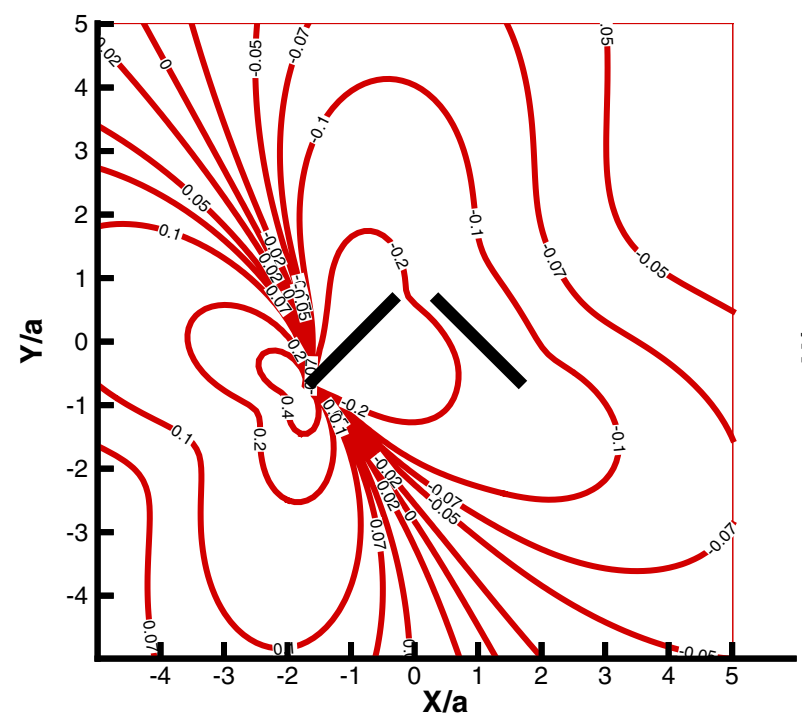

(c)

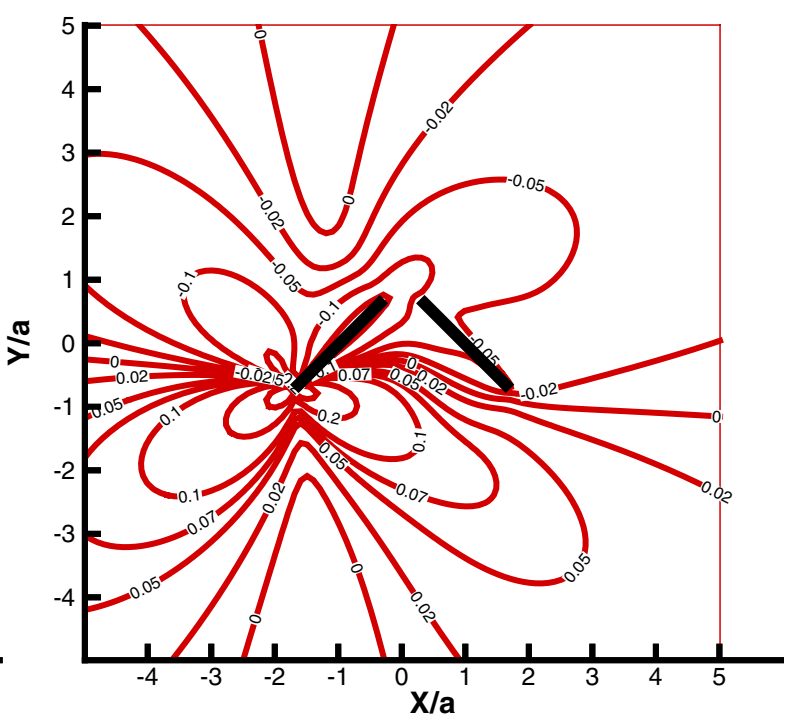

(b)

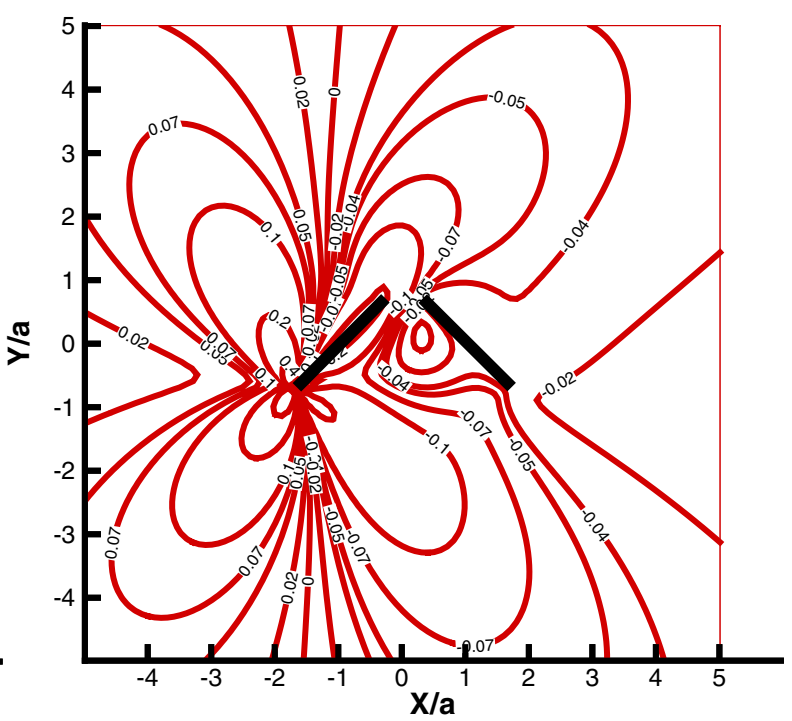

(d)

Figure 14: Variation of the SIFs as the function of the location $(\tilde{x}=x / a, \tilde{y}=y / a)$ of the unit line forces for two inclined cracks. The SIFs are measured at the crack tip $A$ in Figure 6 . (a) $\tilde{K}_{I}$ by $\tilde{b}_{x}=1$, (b) $\tilde{K}_{I I}$ by $\tilde{b}_{x}=1$, (c) $\tilde{K}_{I}$ by $\tilde{b}_{y}=1$, (b) $\tilde{K}_{I I}$ by $\tilde{b}_{x}=1$. 


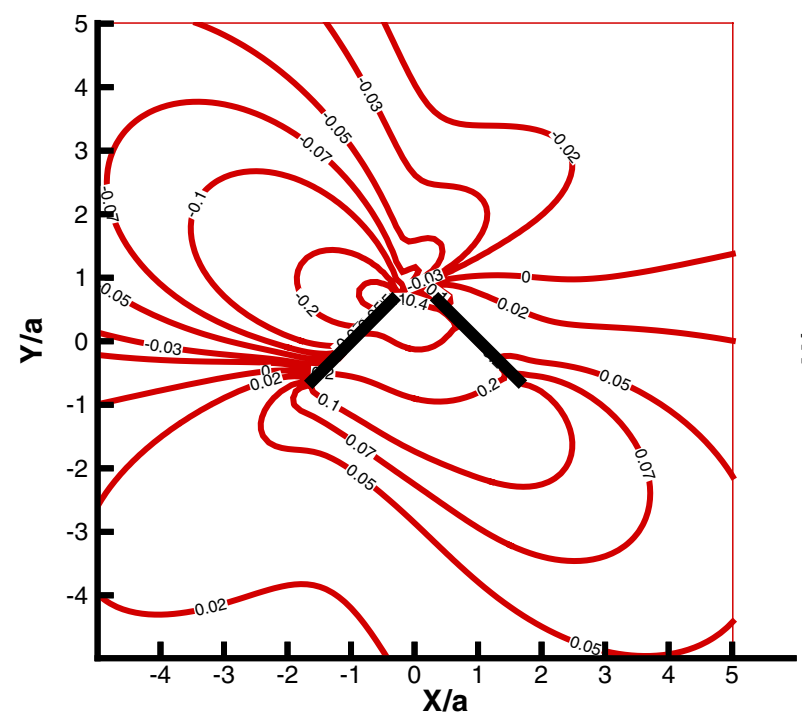

(a)

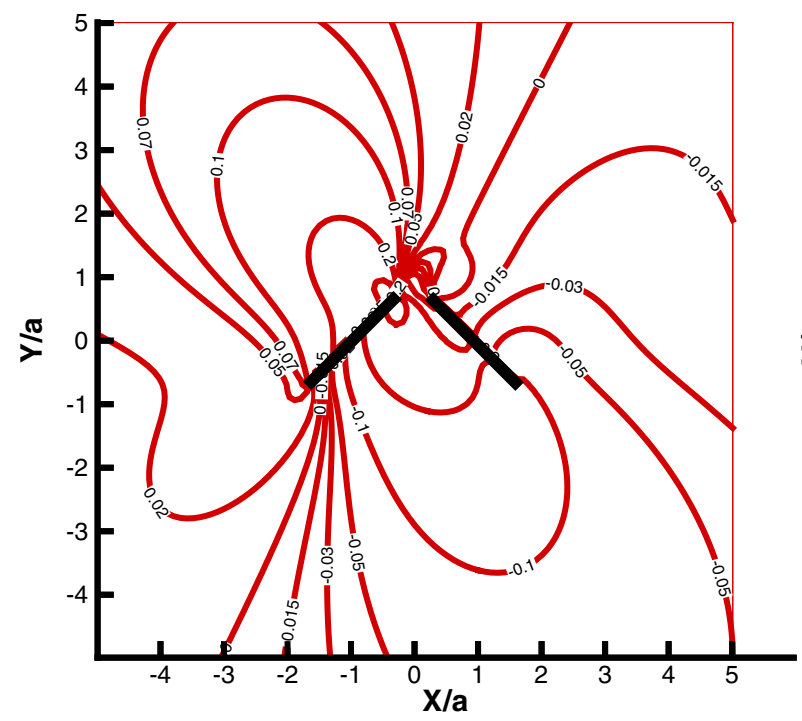

(c)

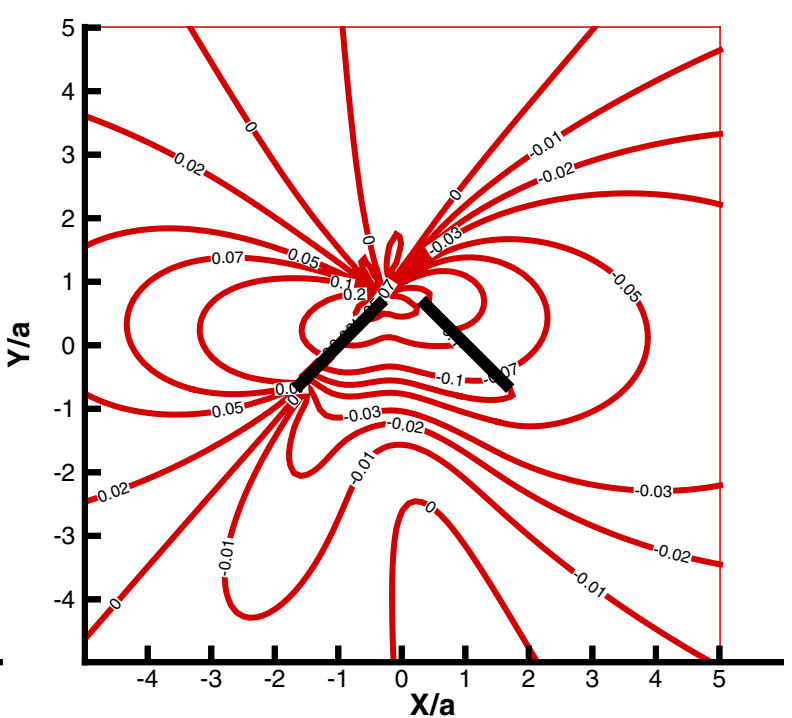

(b)

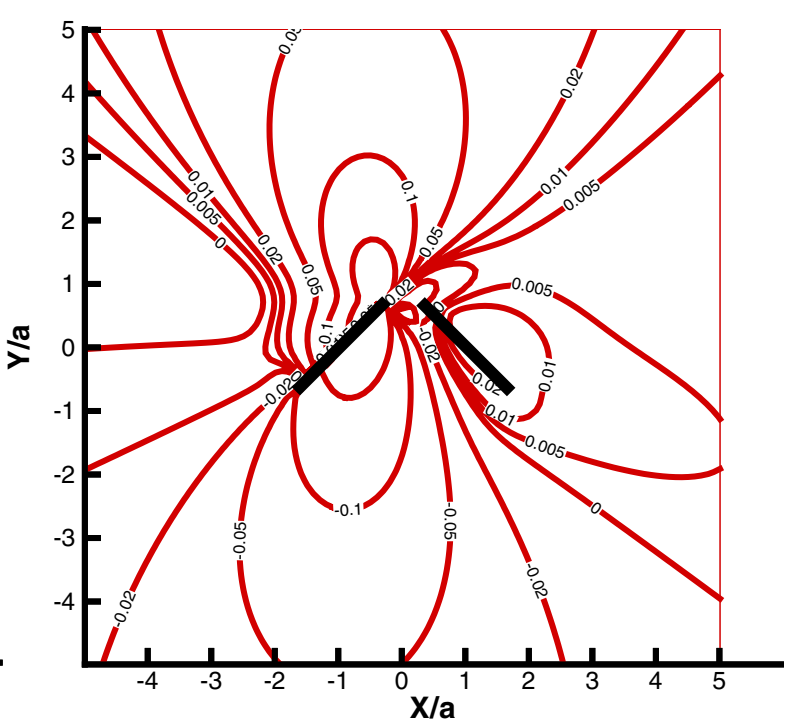

(d)

Figure 15: Variation of the SIFs as the function of the location $(\tilde{x}=x / a, \tilde{y}=y / a)$ of the unit line forces for two inclined cracks. The SIFs are measured at the crack tip $B$ in Figure 6 . (a) $\tilde{K}_{I}$ by $\tilde{f}_{x}=1$, (b) $\tilde{K}_{I I}$ by $\tilde{f}_{x}=1$, (c) $\tilde{K}_{I}$ by $\tilde{f}_{y}=1$, (b) $\tilde{K}_{I I}$ by $\tilde{f}_{x}=1$. 


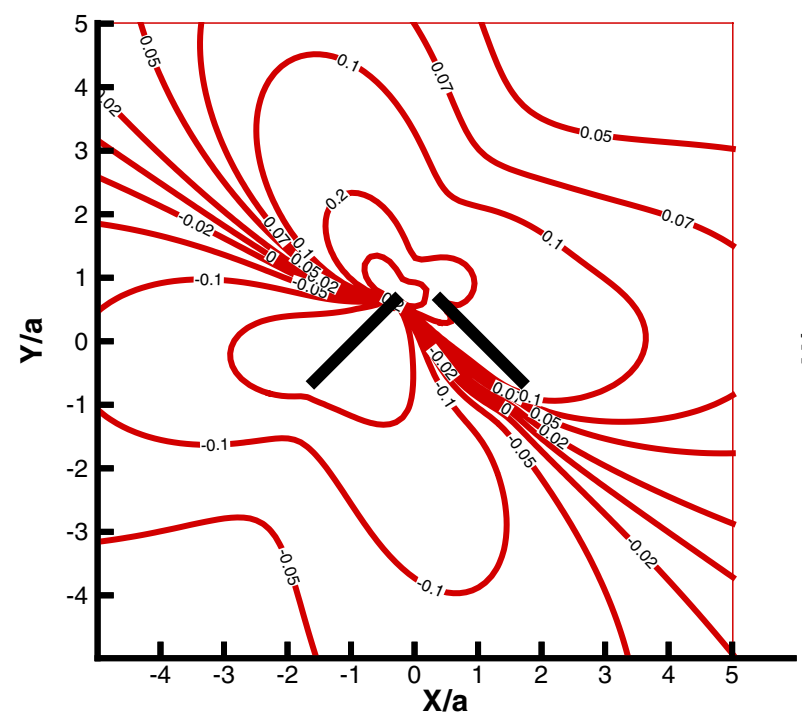

(a)

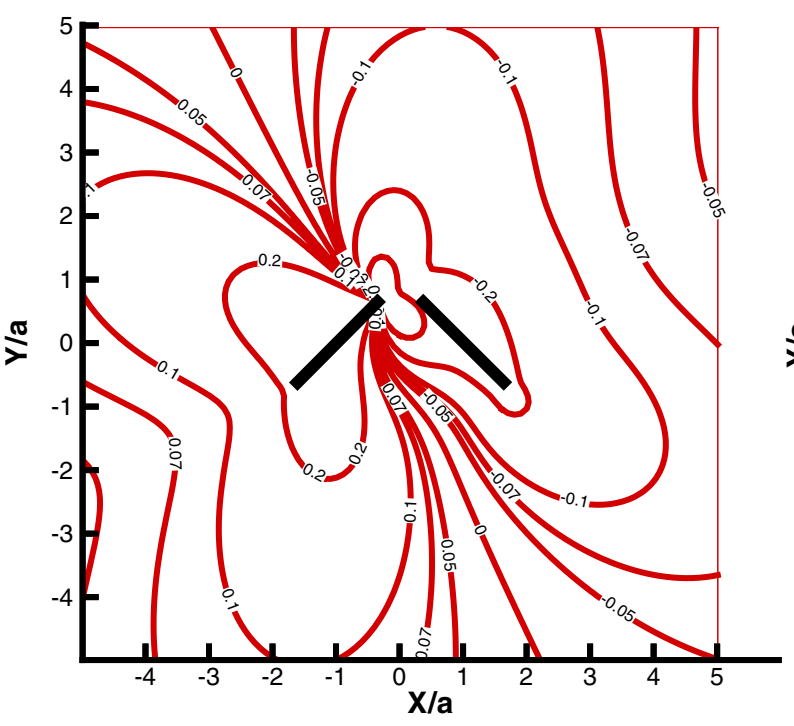

(c)

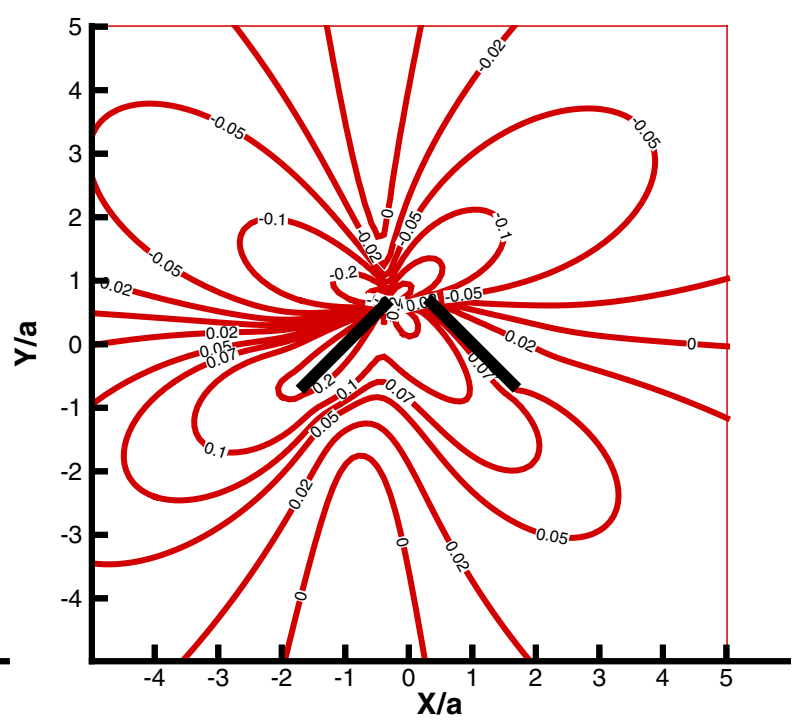

(b)

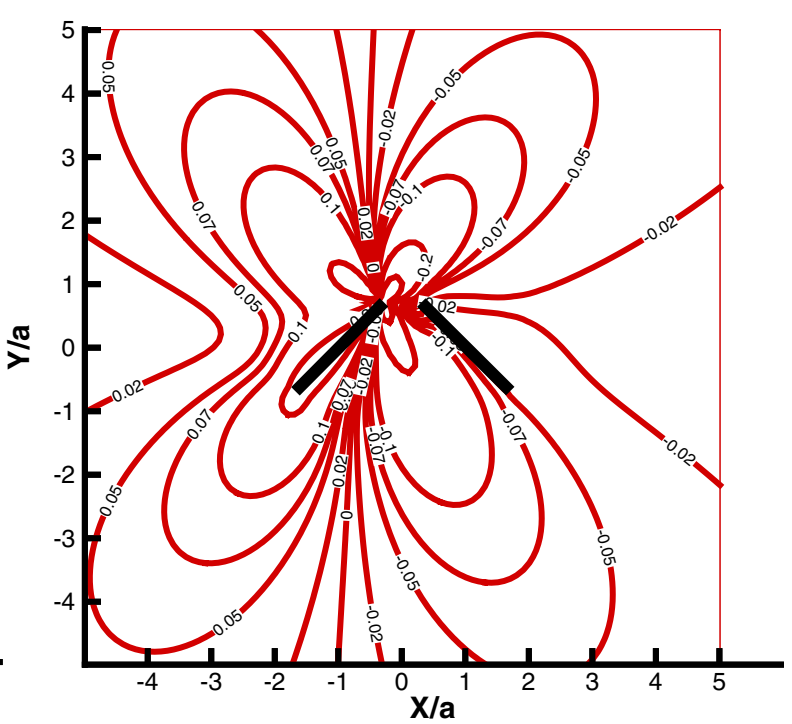

(d)

Figure 16: Variation of the SIFs as the function of the location $(\tilde{x}=x / a, \tilde{y}=y / a)$ of the unit line forces for two inclined cracks. The SIFs are measured at the crack tip $B$ in Figure 6 . (a) $\tilde{K}_{I}$ by $\tilde{b}_{x}=1$, (b) $\tilde{K}_{I I}$ by $\tilde{b}_{x}=1$, (c) $\tilde{K}_{I}$ by $\tilde{b}_{y}=1$, (b) $\tilde{K}_{I I}$ by $\tilde{b}_{x}=1$. 


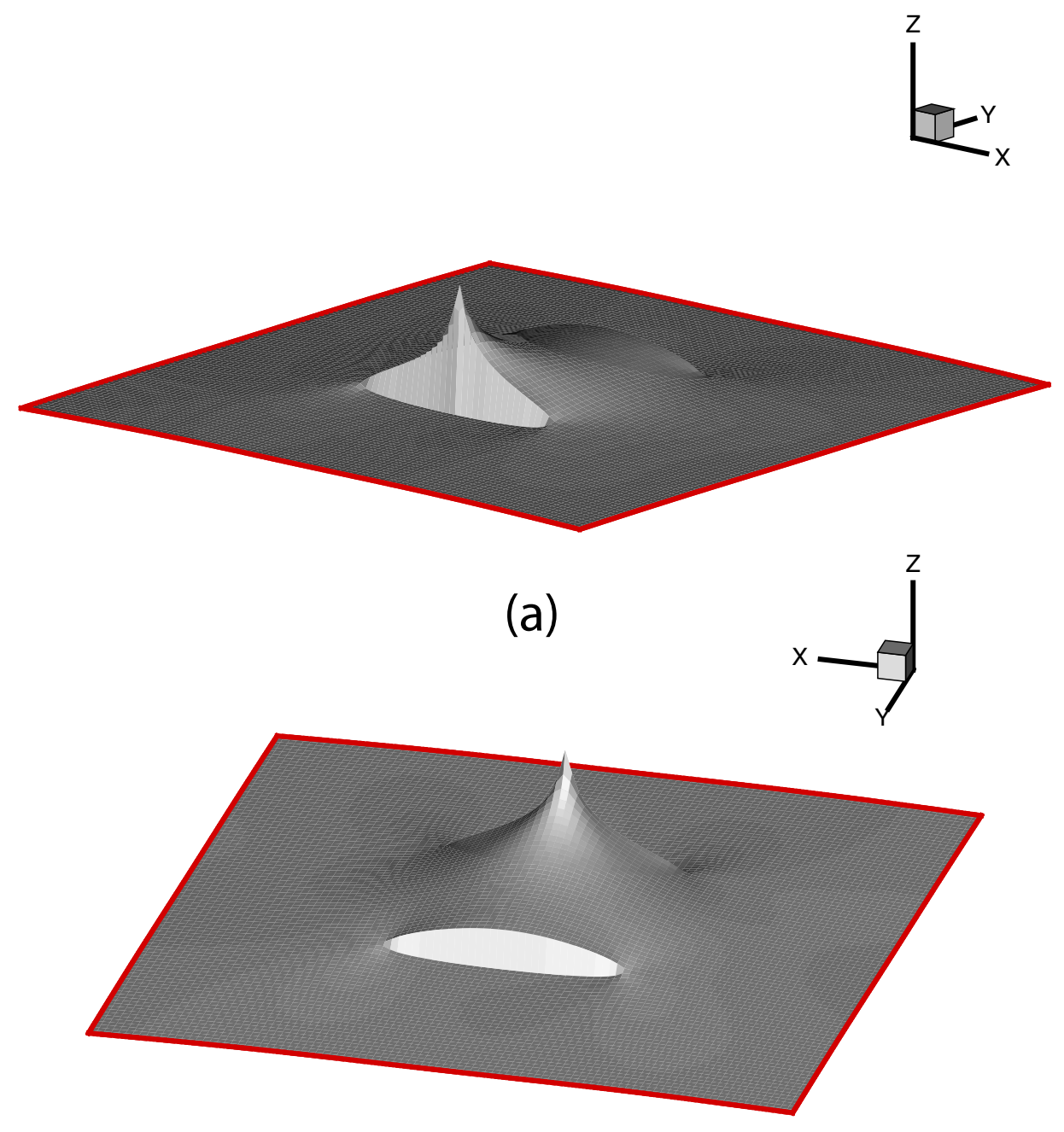

(b)

Figure 17: Two different views of 3-D plots of $\tilde{u}_{y}$ displacement field for the two parallel crack subjected to $\tilde{f}=1.0$ located at the upper lip of the mid point of the lower crack $A B$ in Figure 5. 


\section{References}

[1] J.C.F. Telles, G.S. Castor, and S. Guimarães. A numerical Green's function apparoach for boundary elements applied to fracture mechanics. Int. J. Num. Meth. Engng., 38:3259-3274, 1995.

[2] N.P.P. Silveira, S. Guimarães, and J.C.F. Telles. Accurate hypersingular integral computations in the development of numerical Green's function for fracture mechanics, chapter 6. Singulr Integrals in Boundary Element Methods. Computational Mechanics Publication, Southampton, 1998.

[3] G.S. Castor and J.C.F. Telles. The 3-D BEM implementation of a numerical Green's function for fracture mechanics application. Int. J. Num. Meth. Engng., 48:1199-1214, 2000.

[4] M. Denda and P.R. Quick. Nelectronic journal of boundary elements. 2(1):2268, 2004.

[5] M. Denda and Y. F. Dong. Complex variable approach to the BEM for multiple crack problems. Compt. Methods Appl. Mech. Engrg., Vol. 141:pp. 247-264, 1997.

[6] N.I. Muskhelishvili. Some Basic Problems of the Mathematical Theory of Elasticity. Noordhoff, Groningen, 1958.

\section{Appendix}

\section{A Analytical Green's function for single crack}

\section{A.1 Image term solution}

We consider the line force and dislocation when the infinite domain is cracked over the interval $-a \leq x \leq a$ of the $x$-axis. The Green's function solutions are give in the form

$$
\begin{aligned}
& u^{(\text {total })}=u^{(s)}+u^{(\text {anal })}, \quad t^{(\text {total })}=t^{(s)}+t^{(\text {anal })}, \\
& \sigma^{(\text {total })}=\sigma^{(s)}+\sigma^{(\text {anal })}, \quad \tau^{(\text {total })}=\tau^{(s)}+\tau^{(\text {anal })}
\end{aligned}
$$

for the displacement, traction and stress components consisting of the singular terms $u^{(s)}, t^{(s)}, \sigma^{(s)}$ and $\tau^{(s)}$ and the analytical image terms $u^{(\text {anal })}, t^{(\text {anal })}, \sigma^{(\text {anal })}$ and $\tau^{(\text {anal })}$. Let $w$ and $\rho$ be the images of $z$ and $\xi$ by the mapping function $z=M(w)$ defined by

$$
z=M(w)=R\left(w+\frac{1}{w}\right) ; \quad R=\frac{a}{2}
$$


that maps the crack face into the unit circle and the points $z$ and $\xi$ in the $z$-plane into points $w$ and $\rho$ in the $w$-plane. The image term of the displacement is given by

$$
2 \mu u^{(\text {anal })}(w, \rho)=\kappa \phi_{0}(w, \rho)-\overline{\psi_{0}(w, \rho)}+(\bar{z}-z) \overline{\left(\frac{\phi_{0}^{\prime}(w, \rho)}{M^{\prime}(w)}\right)},
$$

and of the stress by

$$
\begin{aligned}
\sigma^{(\text {anal })}(w, \rho) & =2 \operatorname{Re}\left[\frac{\phi_{0}^{\prime}(w, \rho)}{M^{\prime}(w)}\right] \\
\tau^{(\text {anal })}(w, \rho) & =\frac{\psi_{0}^{\prime}(w, \rho)}{M^{\prime}(w)}-\frac{\phi_{0}^{\prime}(w, \rho)}{M^{\prime}(w)} \\
& +(\bar{z}-z) \frac{\phi_{0}^{\prime \prime}(w, \rho) M^{\prime}(w)-\phi_{0}^{\prime}(w, \rho) M^{\prime \prime}(w)}{\left(M^{\prime}(w)\right)^{3}}
\end{aligned}
$$

where the functions $\phi_{0}(w, \rho)$ and $\psi_{0}(w, \rho)$ are defined by

$$
\begin{aligned}
& \phi_{0}(w, \rho)=\phi_{1}(w, \rho) \gamma+\phi_{2}(w, \rho) \bar{\gamma} \\
& \psi_{0}(w, \rho)=\psi_{1}(w, \rho) \gamma+\psi_{2}(w, \rho) \bar{\gamma}
\end{aligned}
$$

with

$$
\begin{aligned}
\phi_{1}(w, \rho) & =L\left(w, \frac{1}{\rho}\right)+k L\left(w, \frac{1}{\bar{\rho}}\right), \\
\phi_{2}(w, \rho) & =\frac{\bar{\xi}-\xi}{R\left(1-\overline{\rho^{2}}\right)} \frac{1}{w-\frac{1}{\bar{\rho}}}, \\
\psi_{1}(w, \rho) & =\frac{\xi-\bar{\xi}}{R\left(1-\rho^{2}\right)} \frac{1}{w-\frac{1}{\rho}}, \\
\psi_{2}(w, \rho) & =k L\left(w, \frac{1}{\rho}\right)+L\left(w, \frac{1}{\bar{\rho}}\right),
\end{aligned}
$$

and the function $L(w, \eta)$ is defined by

$$
L(w, \eta)=\ln (w-\eta)-\ln w,
$$

with $\eta=\frac{1}{\rho}$ or $\frac{1}{\bar{\rho}}$. The prime attached to the functions indicates the differentiation with respect to $w$. The constant $k$ and the coefficient $\gamma$ are defined by (9). The traction on a line segment with the slope $\theta$ is given by

$$
t^{(a n a l)}(w, \rho ; \theta)=-i\left\{e^{i \theta} \sigma^{(a n a l)}(w, \rho)+e^{-i \theta} \overline{\tau^{(a n a l)}(w, \rho)}\right\} .
$$

\section{A.2 Crack opening displacement and stress intensity factor}

The crack opening displacement $\delta u=u^{+}-u^{-}$is given, from (A.3), by

$$
2 \mu \delta u^{(\text {anal })}=\left\{\kappa\left[\delta L\left(w, \frac{1}{\rho}\right)+k \delta L\left(w, \frac{1}{\bar{\rho}}\right)\right]-\left[\overline{\delta L\left(w, \frac{1}{\bar{\rho}}\right)}+k \overline{\delta L\left(w, \frac{1}{\rho}\right)}\right]\right\} \gamma
$$




$$
+\quad(\kappa+1) \frac{\bar{\xi}-\xi}{R\left(1-\bar{\rho}^{2}\right)} \delta\left(\frac{1}{w-\frac{1}{\bar{\rho}}}\right) \bar{\gamma}
$$

where

$$
\begin{aligned}
& \delta L\left(w, \frac{1}{\rho}\right)=L^{+}\left(w, \frac{1}{\rho}\right)-L^{-}\left(w, \frac{1}{\rho}\right) \\
& \delta L\left(w, \frac{1}{\bar{\rho}}\right)=L^{+}\left(w, \frac{1}{\bar{\rho}}\right)-L^{-}\left(w, \frac{1}{\bar{\rho}}\right)
\end{aligned}
$$

and the function $L(w, \eta)$ for $\eta=1 / \rho, 1 / \bar{\rho}$ is defined by (A.7). The argument $w$ takes the values

$$
w^{ \pm}=\frac{x \pm i \sqrt{a+x} \sqrt{a-x}}{a}
$$

on the upper $(+)$ and lower $(-)$ faces of the crack.

The stress intensity factor, obtained using the stress formula (A.4) along the crack line, is given by

$$
\begin{aligned}
& \left(K_{I}+i K_{I I}\right)^{(\text {anal })}( \pm a) \\
= & 2 \sqrt{\frac{\pi}{a}}\left\{\frac{\bar{\xi}-\xi}{1-\rho^{2}} \frac{1}{\left( \pm 1-\frac{1}{\rho}\right)^{2}} \gamma+\left[k\left(\frac{1}{ \pm 1-\frac{1}{\rho}} \mp 1\right)+\left(\frac{1}{ \pm 1-\frac{1}{\bar{\rho}}} \mp 1\right)\right] \bar{\gamma}\right\},
\end{aligned}
$$

where the sign \pm and $\mp$ follows the location $\pm a$ of the crack tips.

\section{B Whole crack singular element}

According to Denda and Dong [5], the displacement contribution of the whole crack singular element is given by

$$
u=\sum_{m=1}^{M}\left(K^{(m)}(Z) \delta^{(m)}+\overline{L^{(m)}(Z) \delta^{(m)}}\right),
$$

where

$$
\begin{aligned}
K^{(m)}(Z) & =\frac{i}{2(\kappa+1)}\left\{\kappa T^{(m)}(Z)-\overline{T^{(m)}(Z)}\right\} \\
L^{(m)}(Z) & =\frac{i}{2(\kappa+1)}(Z-\bar{Z}) m U^{(m-1)}(Z),
\end{aligned}
$$

with

$$
\begin{aligned}
T^{(m)}(Z) & =\left(Z-\sqrt{Z^{2}-1}\right)^{m} \quad(m \geq 0), \\
U^{(m-1)}(Z) & =-\frac{\left(Z-\sqrt{Z^{2}-1}\right)^{m}}{\sqrt{Z^{2}-1}} \quad(m \geq 0) .
\end{aligned}
$$


The stress contribution is given by

$$
\begin{aligned}
\sigma & =\frac{2 \mu}{a(\kappa+1)} \operatorname{Re}\left\{i \sum_{m=1}^{M} m \delta^{(m)} U^{(m-1)}(Z)\right\}, \\
\tau & =-\frac{i \mu}{a(\kappa+1)} \sum_{m=1}^{M}\left[+m\left(\delta^{(m)}+\overline{\delta^{(m)}}\right) U^{(m-1)}(Z)\right. \\
& \left.+m \delta^{(m)} \frac{Z-\bar{Z}}{Z^{2}-1}\left\{(m+1) T^{(m)}(Z)-U^{(m)}(Z)\right\}\right] .
\end{aligned}
$$

The traction on the line segment with the slope $\theta$ is given by

$$
t=\sum_{m=1}^{M}\left\{K^{*(m)}(Z, \theta) \delta^{(m)}+\overline{L^{*(m)}(Z, \theta) \delta^{(m)}}\right\}
$$

where

$$
\begin{aligned}
K^{*(m)}(Z, \theta) & =\frac{\mu m}{a(\kappa+1)}\left\{e^{i \theta} U^{(m-1)}(Z)+e^{-i \theta} \overline{U^{(m-1)}(Z)}\right\} \\
L^{*(m)}(Z, \theta) & =\frac{\mu m}{a(\kappa+1)}\left\{\left(e^{i \theta}-e^{-i \theta}\right) U^{(m-1)}(Z)\right. \\
& \left.+\quad+e^{i \theta} \frac{Z-\bar{Z}}{Z^{2}-1}\left[(m+1) T^{(m)}(Z)-U^{(m)}(Z)\right]\right\} .
\end{aligned}
$$

Evaluate (B.5) along the crack line to get the traction

$$
t^{+}=\frac{2 \mu}{a(\kappa+1)} \sum_{m=1}^{M} m \delta^{(m)} U_{m-1}(X) \quad(|X| \leq 1),
$$

on the upper crack surface and the stress on the $X$-axis outside the crack

$$
\sigma_{y y}+i \sigma_{x y}= \pm \frac{2 \mu i}{a(\kappa+1)} \sum_{m=1}^{M} m \overline{\delta^{(m)}} \frac{\left(X \mp \sqrt{X^{2}-1}\right)^{m}}{\sqrt{X^{2}-1}},
$$

where the upper and lower signs correspond to $X>1$ and $X<-1$, respectively. The stress intensity factor is extracted, from (B.8), as

$$
K( \pm 1)=K_{I}( \pm 1)+i K_{I I}( \pm 1)=\frac{2 \mu i}{\kappa+1} \sqrt{\frac{\pi}{a}} \sum_{m=1}^{M}( \pm 1)^{m+1} m \overline{\delta^{(m)}}
$$

where $K_{I}$ and $K_{I I}$ are the Mode I and II stress intensity factors in the local $O$-xy coordinate system. 